\title{
The Flare-dominated Accretion Mode of a Radio-bright Candidate Transitional Millisecond Pulsar
}

\author{
Kwan-Lok Li ${ }^{1,2}$ (1) , Jay Strader ${ }^{1}$ (i), James C. A. Miller-Jones ${ }^{3}$ (i), Craig O. Heinke ${ }^{4}$ (i), and Laura Chomiuk ${ }^{1}$ (1) \\ ${ }^{1}$ Center for Data Intensive and Time Domain Astronomy, Department of Physics and Astronomy, Michigan State University, East Lansing, MI 48824, USA \\ lilirayhk@gmail.com \\ ${ }^{2}$ Institute of Astronomy, National Tsing Hua University, Hsinchu 30013, Taiwan \\ ${ }^{3}$ International Centre for Radio Astronomy Research, Curtin University, GPO Box U1987, Perth, WA 6845, Australia \\ ${ }^{4}$ Department of Physics, University of Alberta, CCIS 4-183, Edmonton, AB T6G 2E1, Canada \\ Received 2020 February 26; revised 2020 April 23; accepted 2020 April 29; published 2020 June 1
}

\begin{abstract}
We report new simultaneous X-ray and radio continuum observations of 3FGL J0427.9-6704, a candidate member of the enigmatic class of transitional millisecond pulsars. These XMM-Newton and Australia Telescope Compact Array observations of this nearly edge-on, eclipsing low-mass X-ray binary were taken in the subluminous disk state at an X-ray luminosity of $\sim 10^{33}(\mathrm{~d} / 2.3 \mathrm{kpc})^{2} \mathrm{erg} \mathrm{s}^{-1}$. Unlike the few well-studied transitional millisecond pulsars, which spend most of their disk state in a characteristic high or low accretion mode with occasional flares, 3FGL J0427.9-6704 stayed in the flare mode for the entire X-ray observation of $\sim 20 \mathrm{hr}$, with the brightest flares reaching $\sim 2 \times 10^{34} \mathrm{erg} \mathrm{s}^{-1}$. The source continuously exhibited flaring activity on timescales of $\sim 10-100 \mathrm{~s}$ in both the X-ray and optical/ultraviolet (UV). No measurable time delay between the X-ray and optical/UV flares is observed, but the optical/UV flares last longer, and the relative amplitudes of the X-ray and optical/UV flares show a large scatter. The X-ray spectrum can be well-fit with a partially absorbed power law ( $\Gamma \sim 1.4-1.5$ ), perhaps due to the edge-on viewing angle. Modestly variable radio continuum emission is present at all epochs, and is not eclipsed by the secondary, consistent with the presence of a steady radio outflow or jet. The simultaneous radio/X-ray luminosity ratio of 3FGL J0427.9-6704 is higher than any known transitional millisecond pulsars and comparable to that of stellar-mass black holes of the same X-ray luminosity, providing additional evidence that some neutron stars can be as radio-loud as black holes.
\end{abstract}

Unified Astronomy Thesaurus concepts: Low-mass x-ray binary stars (939); High energy astrophysics (739); Binary pulsars (153)

\section{Introduction}

Transitional millisecond pulsars (tMSPs) are a new sub-class of neutron star low-mass X-ray binaries (NS-LMXBs) that have become observable over the last decade (see Jaodand et al. 2018 for the time-line of some of the most significant events of this class). Unlike typical accreting millisecond X-ray pulsars (AMXPs), these systems switch between distinct states of being a pulsar and an LMXB on timescales that range from weeks to $\sim 10+$ yr (Bond et al. 2002; Thorstensen \& Armstrong 2005; Archibald et al. 2009; Wang et al. 2009; Papitto et al. 2013; Patruno et al. 2014). As the only known bridge between the radio MSPs and LMXBs, they are widely linked to the standard recycling scenario of neutron stars (Alpar et al. 1982; Radhakrishnan \& Srinivasan 1982).

To date, only three tMSPs are known: PSR J1824-2452I in M28 (a.k.a. M28I; Papitto et al. 2013), PSR J1023+0038 (Archibald et al. 2009; Patruno et al. 2014), and PSR J1227 -4853 (Roy et al. 2015). They are all identified as "redback" eclipsing millisecond pulsar binaries, in which the MSP is ablating the low-mass companion (median mass of $0.36 M_{\odot}$; Strader et al. 2019) in a compact orbit (orbital periods of $\lesssim 1$ day).

M28I is currently the only known tMSP that has shown the typical X-ray outburst of AMXPs (i.e., $L_{x} \gtrsim 10^{36} \mathrm{erg} \mathrm{s}^{-1}$ ). In PSRs J1023+0038 and J1227-4853, the accretion state is about two orders of magnitude lower $\left(L_{x} \lesssim 10^{34} \mathrm{erg} \mathrm{s}^{-1}\right)$. In this so-called sub-luminous disk state, at least three accretion modes, namely the low (a few $\times 10^{32} \mathrm{erg} \mathrm{s}^{-1}$ ), high (a few $\left.\times 10^{33} \mathrm{erg} \mathrm{s}^{-1}\right)$, and flare modes $\left(\sim 10^{34} \mathrm{erg} \mathrm{s}^{-1}\right)$ are observed (de Martino et al. 2013; Bogdanov et al. 2015). At least one candidate tMSP, 3FGL J1544.6-1125, has been identified via its display of similar accretion modes and its other optical properties (Bogdanov \& Halpern 2015; Britt et al. 2017). Like 3FGL J1544.6-1125, PSR J1023+0038 and PSR J1227 -4853 have been observed to emit $\mathrm{GeV} \gamma$-rays in the subluminous disk state (Stappers et al. 2014; Johnson et al. 2015). Interestingly, PSR J1023+0038 also exhibited optical pulsations during the sub-luminous disk state, which makes it the first millisecond pulsar ever detected in the optical (Ambrosino et al. 2017). Papitto et al. (2019) argued that the pulsed optical emission originates neither from magnetically channelled accretion nor a rotation-powered pulsar magnetosphere, but synchrotron emission from the intrabinary shock between the pulsar wind and the accretion disk. This would imply that the rotation-powered activity of a pulsar persists in the subluminous disk state.

PSRs J1023+0038 and J1227-4853 are known to spend most of the time in the high (e.g., about 70\% for PSR J1023 +0038 ) and low (about 20\%) modes during the sub-luminous disk state. The mode can promptly switch from high to low in just $\sim 10 \mathrm{~s}$, and then switch back equally rapidly after $100-1000 \mathrm{~s}$ in the low mode. X-ray pulsations have only been detected in the high mode (Papitto et al. 2015; Archibald et al. 2015). PSR J1023+0038 has also been known to enter an extended flare mode occasionally. These extended flaring episodes can last up to $\approx 10 \mathrm{hr}$ in X-rays (Tendulkar et al. 2014; $\mathrm{Li}$ et al. 2014) and $\approx 14 \mathrm{hr}$ in the optical (Papitto et al. 2018). Optical pulsations were also detected when PSR J1023+0038 
was in the flare mode (Papitto et al. 2019), suggesting that the flares are occurring near the neutron star. Recently, Coti Zelati et al. (2019) found another tMSP candidate, CXOU J110926.4650224 , to flare in X-rays for up to $4.5 \mathrm{hr}$. Its identity, however, remains questionable because of its insignificant $\mathrm{GeV} \gamma$-ray counterpart (Abdollahi et al. 2020; Hui \& Li 2019).

In the radio band, the steep spectrum emission and radio pulsations that were clearly detected in the pulsar state disappear in the sub-luminous disk state (Archibald et al. 2009; Papitto et al. 2013; Stappers et al. 2014). Instead, flatspectrum radio emission is detected (Hill et al. 2011; Deller et al. 2015). Recently, Bogdanov et al. (2018) found a strong anti-correlation between the radio and X-ray emission in simultaneous radio and X-ray observations of PSR J1023 +0038: the radio source is bright in the low mode, and faint in the higher mode. These data suggest that a synchrotronemitting outflow is launched during the low mode.

The subject of this paper, 3FGL J0427.9-6704, is a $\gamma$-ray-loud LMXB with an a $8.8 \mathrm{hr}$ orbital period and active accretion, making it very likely to be another sub-luminous tMSP (Strader et al. 2016; S16 hereafter). Uniquely among the members of the class, it is a nearly edge-on system with an inclination of $i \approx 80^{\circ}$. Since spectral lines from both the secondary and disk are visible, 3FGL J0427.9-6704 is essentially an eclipsing doubled-lined spectroscopic binary, which allowed S16 to measure the masses of both the accreting primary $\left(M_{1} \approx 1.8 M_{\odot}\right)$ and secondary $\left(M_{2} \approx\right.$ $\left.0.6 M_{\odot}\right)$.

3FGL J0427.9-6704 was observed in X-rays (3-79 keV) by NuSTAR in 2016. During the $84 \mathrm{ks}$ observations, a nonthermal hard X-ray source was detected, accompanied by three X-ray eclipses. The observation also found strong X-ray variability on timescales of hundreds of seconds, reminiscent of the modeswitching phenomenon seen in tMSPs. In this paper, we report the results of the follow-up XMM-Newton and Australia Telescope Compact Array (ATCA) observations of 3FGL J0427.9-6704, from which we conclude that it has X-ray and optical/ultraviolet (UV) flaring properties distinct from those observed in other tMSPs.

\section{Observations and Data Reduction}

\subsection{XMM-Newton}

We carried out a $77.5 \mathrm{ks}$ XMM-Newton observation (ObsID: 0801650301; PI: J. Strader), starting from 2017 May 2 at 16:14:17 UT to May 3 at 13:45:57 UT. During the observation, all three European Photon Imaging Cameras (EPIC: pn, MOS 1, and MOS 2) were operated under full frame mode (i.e., PrimeFullWindow mode) with time resolutions of $73.4 \mathrm{~ms}$ and $2.6 \mathrm{~s}$ for the $\mathrm{pn}$ and $\operatorname{MOS} 1 / 2$ cameras, respectively. The Medium1 filter was used to optimize the X-ray data quality. For the Optical/UV Monitor Telescope (OM), fast mode (time resolution of $0.5 \mathrm{~s}$ ) with a "white" filter (effective wavelength at $406 \mathrm{~nm}$ with a $347 \mathrm{~nm}$ bandpass width) was used to obtain high-speed optical/UV photometry of the system. We did not use the two reflection grating spectrometers owing to the faintness of the source.

We applied the standard analysis tools in the Science Analysis System (SAS; version 15.0.0) and HEAsoft (version 6.22) with the calibration files obtained from the on-line cifbuild server ${ }^{5}$ to reduce and analyze the XMM-Newton data. The SAS task xmmextractor was used for EPIC's

\footnotetext{
https://www.cosmos.esa.int/web/xmm-newton/cifbuild
}
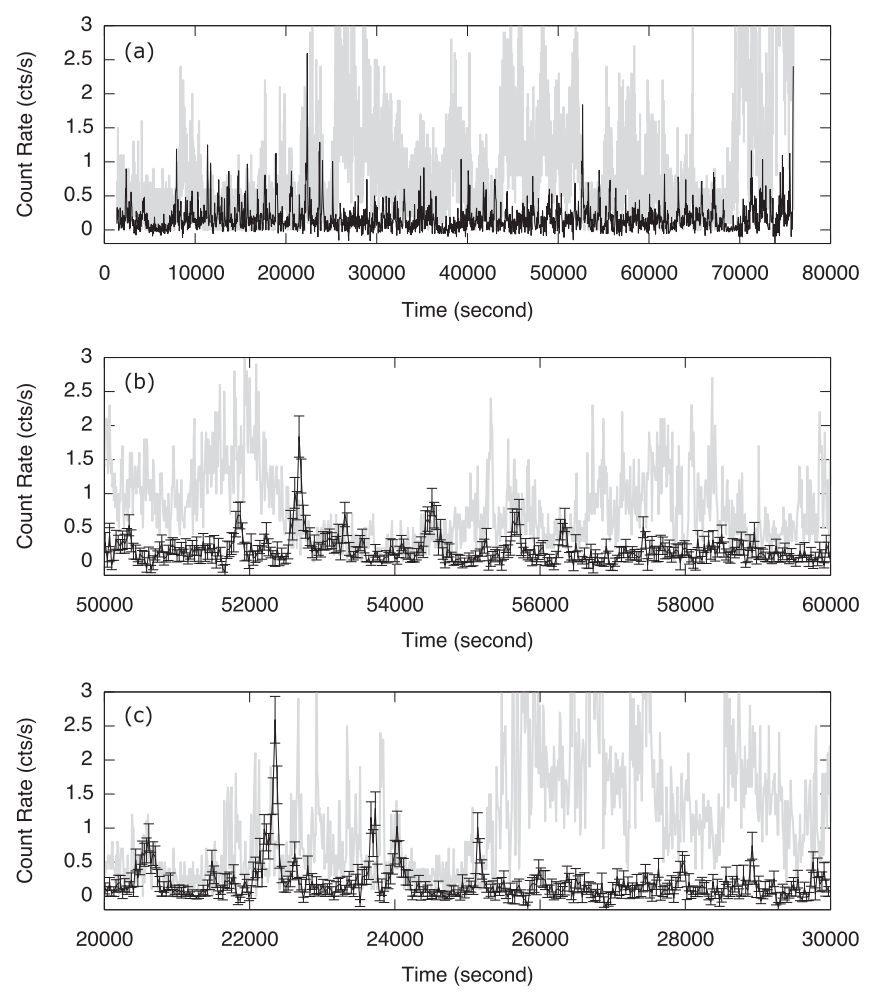

Figure 1. (a) Black curve: reduced EPIC light curve of 3FGL J0427.9-6704 (pn + MOS 1/2); gray curve: flaring background obtained by pn. (b, c) Two arbitrary zoomed-in views of (a) with $1 \sigma$ uncertainties of the EPIC light curve. No correlation is found between the two curves.

reduction processes, with standard filtering (\#XMMEA_EP \& \& $\mathrm{FLAG}==0 \quad \& \&$ PATTERN $\leqslant 4$ for $\mathrm{pn} ;$ and \#XMMEA_EM $\& \&$ $F L A G==0 \& \&$ PATTERN $\leqslant 12$ for MOS 1/2). To deal with the high flaring particle background, exposure periods with count rates of the pattern zero events higher than 0.4 counts $\mathrm{s}^{-1}$ in $10-12 \mathrm{keV}$ for $\mathrm{pn}$ and 0.35 counts $\mathrm{s}^{-1}$ in $>10 \mathrm{keV}$ for MOS $1 / 2$ (recommended values in the XMM-Newton user manual) were removed in the imaging and spectral analyses. The flaring background heavily contaminated the pn observation, with an effective exposure time of $24 \mathrm{ks}$ left after the filtering. Fortunately, the MOS $1 / 2$ observations were less affected, with an effective exposure time $70 \mathrm{ks}$ for each MOS. For the extraction regions, circular source regions with optimum radii determined by eregionanalyse were used $\left(r=26^{\prime \prime}, 24^{\prime \prime}\right.$, and $27^{\prime \prime}$ for pn, MOS 1, and MOS 2, respectively), while source-free annulus regions with inner/outer radii of $60^{\prime \prime} / 70^{\prime \prime}$ were used for the background. The full energy band of $0.2-10 \mathrm{keV}$ was used in the analysis, unless mentioned otherwise. In Figure 1, we plotted the background-subtracted light curves of 3FGL J0427.9-6704 together with the pn flaring background to examine whether the flaring background can contaminate the reduced light curve significantly. It is clearly shown in the figures that the effect is very minor and therefore the whole period of data will be used in our lightcurve analysis.

The OM images and light curves were reduced and extracted by omfchain. There were 48 exposure segments taken in a $22 \times 23$ pixel OM window (plate scale of about 0 ". 48 per pixel). Except for the last one with $2280 \mathrm{~s}$, every segment had an exposure time of $1200 \mathrm{~s}$ with a $\sim 300 \mathrm{~s}$ observing gap (there was one exception before the last second exposure, which had a gap of $\sim 2000 \mathrm{~s})$. Two sources, the optical counterpart of 
3FGL J0427.9-6704 and a faint non-variable source (5!" 5 to the northeast of 3FGL J0427.9-6704, about 6 times fainter than the target), were detected and well-resolved from each other. Aperture photometry with an aperture size of 6 pixels in radius (about $3 \mathrm{FWHM}$ in diameter) was applied to extract the OM light curves of 3FGL J0427.9-6704. An annulus with inner/outer radii of 7.2 and 15 pixels was used as the background region, in which the back/foreground faint source was excluded with a 6 pixel radius circular region. All times are presented in the frame of Barycentric Dynamical Time, converted by the SAS task barycen with the JPL solar system ephemeris DE200 (Standish 1990).

\subsection{Australia Telescope Compact Array}

We arranged strictly simultaneous radio observations of 3FGL J0427.9-6704 using ATCA, observing for as long as the source was above the horizon, from 18:10 UT on 2017 May 2 to 13:07 UT on 2017 May 3, under project codes CX364 and $\mathrm{C} 3170$. The array was in the extended 6A configuration, with all six antennas aligned east-west, with a maximum baseline of $6 \mathrm{~km}$. We used the Compact Array Broadband Backend (Wilson et al. 2011) to observe simultaneously at central frequencies of 5.5 and $9.0 \mathrm{GHz}$, with $2048 \mathrm{MHz}$ of bandwidth in each of the two frequency bands.

We used the standard extragalactic calibrator PKS B1934 -638 as a bandpass calibrator and to set the flux density scale, and the nearby sources $0355-669$ and then J0425 -6646 (after the former calibrator set) to determine the complex gain solutions. We reduced the data using standard procedures within the Common Astronomy Software Application (McMullin et al. 2007). We imaged the calibrated data using Briggs weighting with a robust parameter of 0.5 , as a compromise between sensitivity and minimizing sidelobe levels. The source was detected at high significance in both frequency bands.

We imaged several subsets of data to determine whether the radio emission changed when in eclipse, and how it varied with the changing X-ray count rates defined in Section 3.2. Finally, we made time-resolved light curves. Since the array was in an east-west configuration, the instantaneous $u v$-coverage was linear, and not suitable for imaging. We therefore subtracted out all other sources in the field, using our best image made from the entire data set at each frequency, leaving only 3FGL J0427.9-6704 in the visibility data, which we fit in the $u v$-plane using a point source model fixed at the known source position. We found that 10 minute bins provided the best compromise between time resolution and sensitivity.

\subsubsection{Data}

We previously obtained ATCA radio data of 3FGL J0427.9 -6704 from 22:29 UT on 2016 August 27 to 04:38 UT on 2016 August 28, under project code CX365 and in the extended $6 \mathrm{C}$ configuration. The receiver setup, calibrators, and imaging were the same as for the 2017 data. A single image was made at each of the central frequencies of 5.5 and $9.0 \mathrm{GHz}$. These radio data were not simultaneous with any X-ray observations.

\section{X-Ray and Optical/UV Results}

\subsection{Light Curves}

3FGL J0427.9-6704 was clearly detected in X-rays (EPIC) and optical/UV (OM), with net count rates of 0.2 counts $\mathrm{s}^{-1}$ $(\mathrm{pn}+\operatorname{MOS} 1 / 2)$ and 6 counts $\mathrm{s}^{-1}$, respectively (Figures 2 and 3 ). Both light curves are dominated by strong flares on timescales of $\sim 10-100 \mathrm{~s}$. In addition, each light curve shows three eclipses, which perfectly match the orbital ephemeris presented in S16 (Figures 2 and 3). During the eclipse phases, the X-ray and optical fluxes are lower, and the flaring phenomenon is not observed. We tested both light curves for periodic signals longer than $1 \mathrm{~s}$ using powspec, with no clear periodicity found in the frequency range $10^{-5}-1 \mathrm{~Hz}$.

\subsubsection{Flare Properties}

Here we discuss the detailed properties of the flares. Except for those X-ray flares detected during the OM observing gaps, almost every X-ray flare has an optical/UV counterpart. The converse is not true: some optical/UV flares are not detected in the X-rays (e.g., the flare at $t \approx 58,000 \mathrm{~s}$ in Figure 2).

As a simple initial model for the flares, we fit every individual flare detected by EPIC and OM with a Gaussian. In the fitting, we did not include the data in the eclipses, and simply assumed a constant baseline as the "quiescent" emission. While the choice of Gaussian is somewhat arbitrary, it turns out that this model can describe the flares reasonably well (see Figures 2 and 3).

For the EPIC data, the light curve was binned in $30 \mathrm{~s}$ intervals to obtain a good balance between the signal-to-noise and the timing resolution. Using an initial guess of 0.1 counts $\mathrm{s}^{-1}$ as the baseline, we iteratively found new flares and re-fitted the light curve until no data point exceeded the model by $2 \sigma$. The same algorithm was also applied for the OM light curve, but a smaller binning factor of $20 \mathrm{~s}$ and a higher initial baseline of 4 counts s $\mathrm{s}^{-1}$ were adopted. A higher detection threshold of $4.5 \sigma$ was also used to avoid over-fitting. Otherwise, we found that all residual features were fit with low-amplitude "flares." We emphasize that this technique is not designed to achieve a perfect statistical model of the data, but instead to give a first-order sense of the frequency and amplitude of the flares.

As shown in Figures 2 and 3, the X-ray and optical/UV flares that are visually obvious are all identified by our technique. Outside of eclipse, on average we detect an X-ray (optical/UV) flare every 540 (470) s. The occurrence rate is high compared with PSRs J1023+0038 and J1227-4853, for which the flare occurrence rates are just up to a few tens of events per day on average (de Martino et al. 2013; Bogdanov et al. 2015; Kennedy et al. 2018; Papitto et al. 2018). The flaring state of PSR J1023+0038 observed in the optical occupied about $15.6 \%-22 \%$ of the time during the Kepler K2 observation (Kennedy et al. 2018; Papitto et al. 2018). Using the FWHM durations of the detected OM flares, we found that the flaring fraction is $\approx 43 \%$ for 3FGL J0427.9-6704, which is double the fraction seen in PSR J1023+0038. We show a selection of 16 pairs of flares in Figure 4.

Figure 5 shows the best-fit parameters for each detected flare as a function of orbital phase. The median flare timescale (defined by the best-fit Gaussian sigma) for the X-ray (optical/ UV) flares is $24 \mathrm{~s}(72 \mathrm{~s})$, equivalent to a FWHM of $57 \mathrm{~s}(170 \mathrm{~s})$. These timescales are consistent with some of the fastest flares seen in the two tMSPs (i.e., duration of a few minutes or less), but still significantly shorter than that of the slowest events 

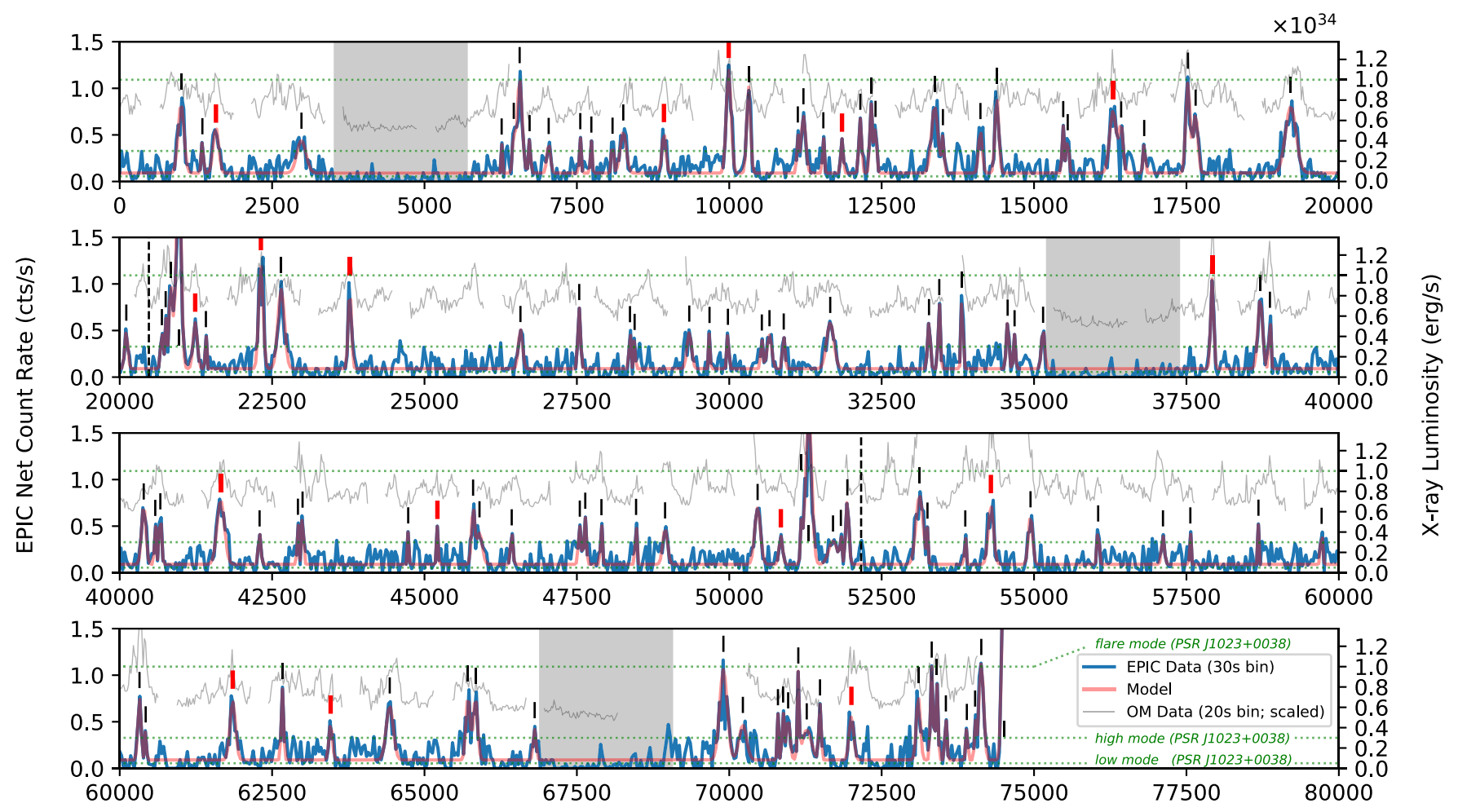

Barycentric Time since BJD (TDB) $=2457876.20508$ (seconds)

Figure 2. X-ray light curve (0.2-10 keV; blue) of 3FGL J0427.9-6704 observed by XMM-Newton in 2017 May. The left $y$-axis shows the corrected count rate measured by pn and MOS 1/2 (the high flaring background intervals are also included), while the right axis refers to the X-ray luminosity inferred from the best-fit partially absorbed power-law model in Averagel (see Table 1 for the best-fit parameters) with $d=2.3 \mathrm{kpc}$. The three $2200 \mathrm{~s}$ eclipses are all indicated by the gray shaded regions based on the solution in S16 while the two vertical dashed lines indicate the inferior conjunction of the binary. The red line is the best-fit Gaussian model for the X-ray flares (see the text for details). Every flare detected is marked with a vertical bar on the top. Red and thicker bars are used to indicate the 16 representative flares (see Figure 4), and the rest are black. The gray narrow curve is the OM light curve scaled to fit the figure (see Figure 3 for the original). The three horizontal green dashed lines represent the X-ray luminosities $(0.3-10 \mathrm{keV})$ of PSR J1023+0038 in the flare $\left(\approx 10^{34} \mathrm{erg} \mathrm{s}^{-1}\right)$, high $\left(\approx 3 \times 10^{33} \mathrm{erg} \mathrm{s}^{-1}\right)$, and low $\left(\approx 5 \times 10^{32} \mathrm{erg} \mathrm{s}^{-1}\right)$ modes, which are obtained from Bogdanov et al. (2015).

observed (i.e., $\approx 45 \mathrm{~min}-14 \mathrm{hr}$; de Martino et al. 2013; Bogdanov et al. 2015; Papitto et al. 2018). The typical uncertainties for the per flare amplitudes and timescales for the EPIC (OM) are 0.3 counts s $^{-1}$ (1.0 counts s $\left.{ }^{-1}\right)$ and $13 \mathrm{~s}(15 \mathrm{~s})$, though the uncertainties for some flares may be underestimated if the assumed model is a poor fit. This figure also shows that there is no significant evidence for a correlation between flare occurrence or properties with orbital phase. The brightest flares reach $L_{X}(0.2-10 \mathrm{keV})$ of $\sim 2 \times 10^{34} \mathrm{erg} \mathrm{s}^{-1}$.

To study the relationship between the X-ray and optical/UV flares, we used the HEAsoft task crosscor to calculate the cross-correlation function between the light curves, parameterizing the time delay of the optical/UV emission as $\Delta t_{d}$. We formally find that the cross-correlation peaks at $\Delta t_{d}=5 \mathrm{~s}$ : the X-rays lead the optical/UV emission by about $5 \mathrm{~s}$. To determine the uncertainty in this value, we simulated $10^{4}$ pairs of light curves based on the EPIC and OM data, and repeated the crosscor calculations with the simulated data. The distribution of the simulated crosscorrelation peaks (Figure 6) gives $\Delta t_{d}=4.5 \pm 6.8 \mathrm{~s} \quad(90 \%$ confidence interval). Hence there is no strong evidence for a time delay of the optical/UV emission compared to the X-ray emission. To put this in context, the light curve modeling of S16 found that the outer edge of the accretion disk is $\sim 0.98 R_{\odot}(\sim 2.3 \mathrm{lt}-\mathrm{s})$ from the neutron star.

In Figure 7 we directly compare the multi-wavelength properties of 62 optical/UV flares, each of which has one and only one detectable X-ray counterpart within \pm 60 s (i.e., equal to two bins of the X-ray light curve shown in Figure 2). Interestingly, most of the flares have longer timescales in optical/UV than in X-rays (e.g., Figure 4(h)), with only five counterexamples found. We checked whether the trend could be affected by the generous $\pm 60 \mathrm{~s}$ matching criterion, by alternatively adopting \pm 10 and $\pm 100 \mathrm{~s}$ as the allowed time offset, but found similar results.

The average optical-to-X-ray timescale ratio is 3.5 (with a standard deviation of 2.4), although the samples do not seem to follow a constant ratio (Figure 7(a)). For the five outliers, three of them have timescale ratios larger than 0.8 with the $\mathrm{X}$-ray timescales just $\lesssim 10 \mathrm{~s}$ longer. Therefore, their timescale ratios could be consistent, considering the per-fit uncertainties. The other two outlier flares have rather low timescale ratios $(\lesssim 0.7)$, and their zoomed-in light curves can be found in Figures 4(o) and (e). The X-ray and optical/UV flare amplitudes are roughly correlated (larger X-ray flares generally lead to larger optical/ UV flares), but with a large scatter (Figure 7(b)). The amplitudes themselves arguably change on short timescales (e.g., Figure 4(f)), which could explain part of this scatter.

\subsection{X-Ray Spectral Analysis}

Since the X-ray data are strongly variable, we separated the data into several groups for independent spectral analyses. 

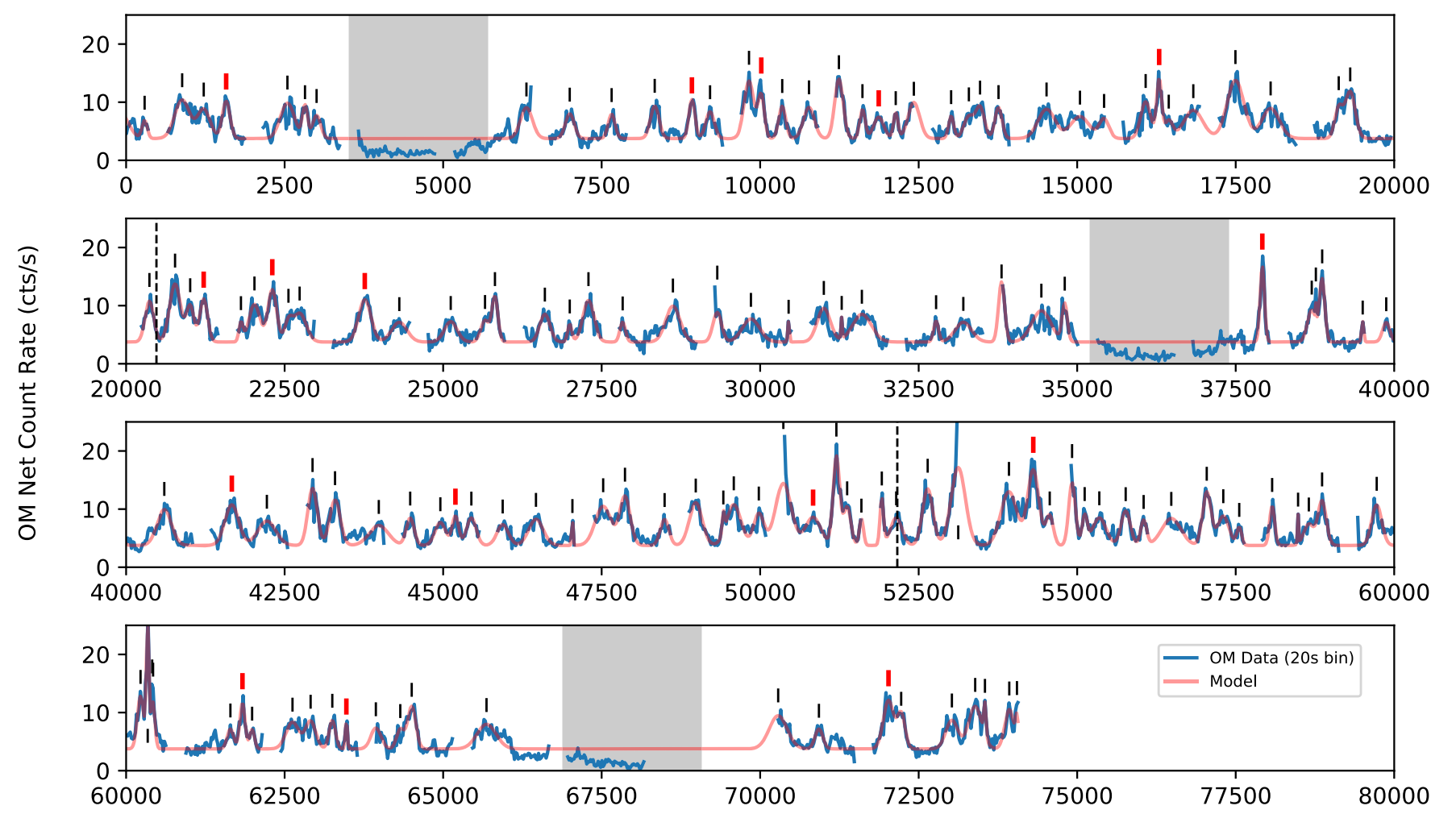

Barycentric Time since BJD (TDB) $=2457876.20508$ (seconds)

Figure 3. Same as in Figure 2, but for the OM optical/UV observations alone.

1. Average1 (MOS 1/2): the out-of-eclipse spectrum, selected based on the orbital timing solution in S16.

2. Highl (MOS 1/2): a subset of Average1, with EPIC net count rates $>0.6$ counts $\mathrm{s}^{-1}$ per $30 \mathrm{~s}$ bin (Figure 2).

3. Mediuml (MOS 1/2): similar to Highl, but with count rates between 0.3 and 0.6 counts $\mathrm{s}^{-1}$.

4. Lowl (MOS 1/2): similar to Highl, but with count rates $\leqslant 0.3$ counts s ${ }^{-1}$.

5. Eclipse (pn and MOS 1/2): data taken during the three eclipse phases.

As mentioned in Section 2.1, the pn and MOS 1/2 data have different time coverages after the flaring background filtering, in which the MOS $1 / 2$ data are less affected. Therefore, only the MOS $1 / 2$ spectra were used in the analysis, except for the Eclipse group, in which the pn data were included. We also prepared four additional data groups with suffix "2" (e.g., Average 2), which contain also the pn spectra. However, the fitting results obtained from these data sets are for reference purposes only, and the following discussions are all based on the primary data sets (suffix " 1 ").

All the X-ray spectral fitting processes were done by XSPEC (version $12.9 .1 \mathrm{~m}$ ). The spectra were binned using grppha to at least 20 counts per bin, and then fit with given spectral models based on a chi-squared statistic $\left(\chi^{2}\right)$, unless otherwise stated. Various spectral models were tested. In these models, two components of hydrogen column density $\left(N_{\mathrm{H}}\right)$ were assumed for the Galactic foreground and intrinsic absorptions. ${ }^{6}$ The Galactic value was fixed to $N_{\mathrm{H}}=3.66 \times 10^{20} \mathrm{~cm}^{-2}$ obtained

\footnotetext{
6 Except for the Eclipse data, as the resultant spectrum does not have a sufficient signal-to-noise ratio $(\mathrm{S} / \mathrm{N})$ for modeling an intrinsic $\mathrm{X}$-ray absorption.
}

from the Leiden/Argentine/Bonn map (Kalberla et al. 2005), while the intrinsic $N_{\mathrm{H}}$ was allowed to vary. In addition, an energy-independent multiplicative factor was employed to the whole physical model to account for the cross-calibrations among the EPIC detectors (see the footnotes in Table 1 for the definitions). Throughout the analysis, all the listed uncertainties were calculated at $90 \%$ confidence level for one interesting parameter (i.e., $\Delta \chi^{2}=2.71$; Avni 1976).

\subsubsection{Average Spectra}

We first tried a simple power-law to gain insights into detailed spectral modeling. The best fit gives a bad fitting statistic of $\chi_{\nu}^{2}=2.4$ (188 dof) with a very hard photon index of $\Gamma \approx 0.5$ and an intrinsic absorption of $N_{\mathrm{H}} \approx 4 \times 10^{22} \mathrm{~cm}^{-2}$. In general, the spectral shape is saddle-like, with a soft X-ray excess below $2 \mathrm{keV}$, which is the primary cause of the bad fit. An additional thermal emission component corresponding to the soft excess was thus considered. Given that the huge intrinsic absorption for the power-law component would have absorbed most of the soft thermal X-rays (if any), we once assumed an independent intrinsic absorption only for the thermal emission (this would be the case if, e.g., the emission regions for the thermal and nonthermal components were different). However, the best-fit value of this extra $N_{\mathrm{H}}$ always goes to zero (e.g., $N_{\mathrm{H}}<2 \times 10^{20} \mathrm{~cm}^{-2}$ for a blackbody fit with the best-fit value found at zero; the same situation was also seen in all other data groups). We therefore removed this absorption component and left the thermal emission solely absorbed by the Galactic foreground medium.

Statistically either a single temperature blackbody (bbodyrad in XSPEC) or a multi-temperature disk blackbody (diskbb) can 

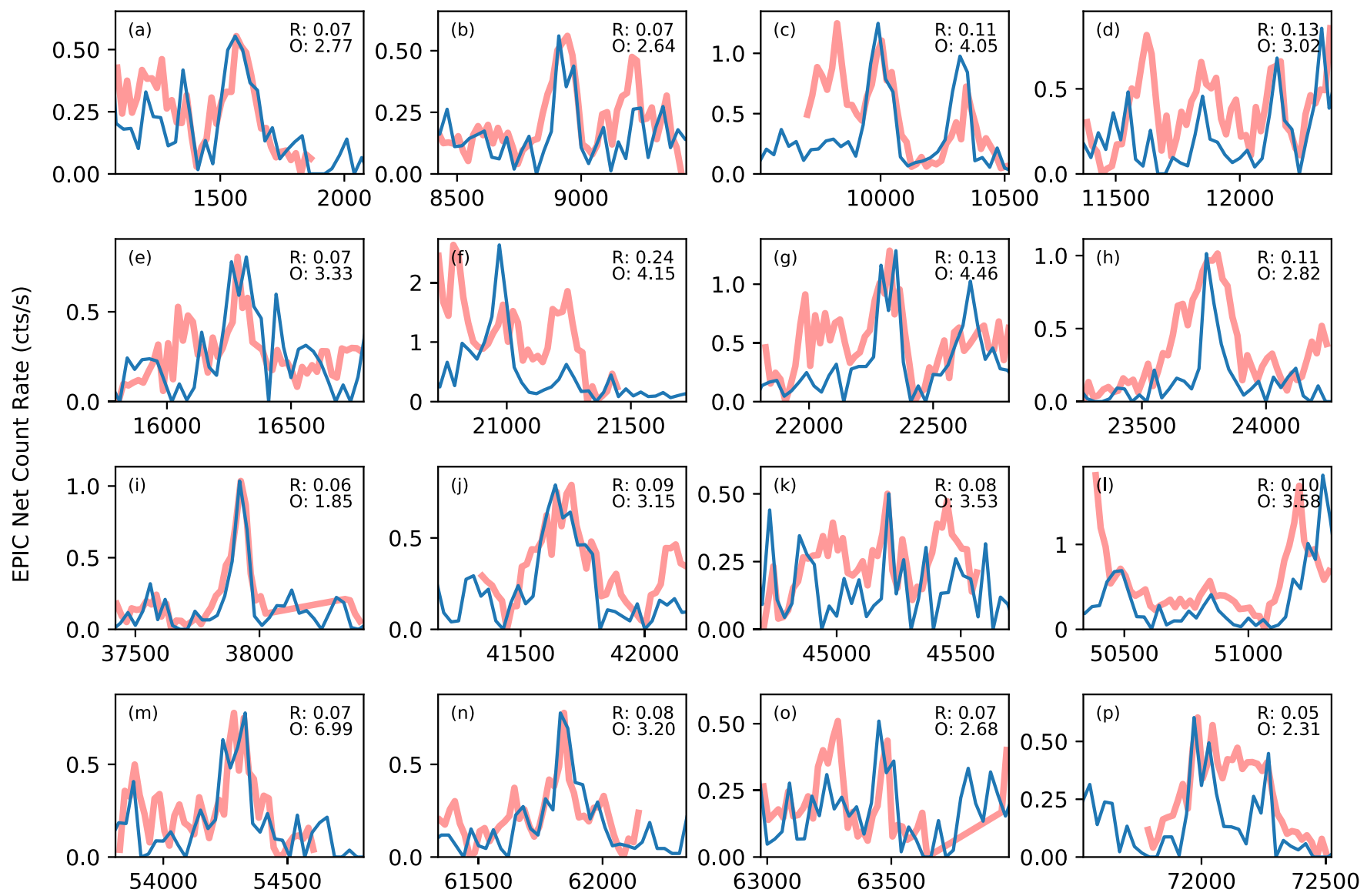

Barycentric Time since BJD (TDB) $=2457876.20508$ (seconds)

Figure 4. EPIC X-ray (blue and thinner) and optical/UV (red and thicker) light curves of 16 arbitrarily chosen flares to show the range of relationships between the $\mathrm{X}$-ray and optical flares. Each zoom-in view is centered at the peak of the chosen flare. The optical/UV light curves shown are scaled linearly to fit the X-ray ones for easier comparison, i.e., $C_{s}=R\left(C_{o}-O\right)$, where $C_{s}$ is the scaled count rate, $C_{o}$ is the original count rate, $R$ is the X-ray-to-optical/UV flare amplitude ratio, and $O$ is the minimum count rate of the original optica/UV light curve in the interval. $R$ and $O$ can be found in the upper right corner of each plot.

improve the power-law fit significantly. The diskbb model is slightly better in terms of $\chi^{2}$ statistics $\left(\chi_{\nu}^{2}=1.17\right.$ and 1.09 for bbodyrad and diskbb, respectively). With a soft thermal component, the best-fit photon index becomes softer (i.e., $\Gamma \approx 0.9-1.1$ ), and comparable to other redback MSP systems (see, e.g., Lee et al. 2018). As expected, the thermal component is very soft with best-fit temperatures of $T_{\mathrm{bb}} \approx 0.3 \mathrm{keV}$ for bbodyrad and $T_{\text {in }} \approx 0.5 \mathrm{keV}$ (the inner disk temperature) for diskbb. Compared with the nonthermal X-ray component, they are very faint. We use the best-fit normalizations and the distance of $d=2.3 \mathrm{kpc}$ estimated by Gaia (Bailer-Jones et al. 2018), which is consistent with the distance from modeling the light curve in S16. The best-fit blackbody emission region is $R_{\mathrm{bb}} \approx 0.2$ $\mathrm{km}$ in radius, and the inner disk radius of the disk is $R_{\text {in }} \approx 0.1 \mathrm{~km}$ ( $\cos i=0.18$ assumed; S16). Apparently, the inner radius is much smaller than the size of a typical neutron star (radius of $\sim 10 \mathrm{~km}$ ), making the diskbb case highly unlikely. We also fit the spectrum with the neutron star atmospheric model, nsa, for more realistic estimates on the temperature and the emission size. In the nsa model, we assumed a non-magnetized (i.e., $B<10^{9} \mathrm{G}$ ) neutron star of $M_{\mathrm{NS}}=1.4 M_{\odot}$ and $R_{\mathrm{NS}}=10 \mathrm{~km}$. The best-fit temperature is lower $\left(T_{\mathrm{nsa}} \approx 0.2 \mathrm{keV}\right)$ and the emission region is significantly bigger $\left(R_{\mathrm{nsa}} \approx 0.5 \mathrm{~km}\right)$.

Besides an additional thermal component, a partial covering fraction absorption (pcfabs) with a simple power law (hereafter called a partially absorbed power law) can also explain the saddleshaped X-ray spectrum very well $\left(\chi_{\nu}^{2}=1.1\right.$ with 187 dof; Figure 8 ). In the best-fit partially absorbed power law, the intrinsic absorber only covers about $97 \%$ of the X-rays in our line of sight, and $3 \%$ of the X-ray emission "leaked" to produce the observed soft X-ray bump. Comparing with the simple power-law models with/without a thermal component, the intrinsic hydrogen column density is significantly higher $\left(N_{\mathrm{H}}=(10.9 \pm 0.5) \times 10^{22} \mathrm{~cm}^{-2}\right)$. The photon index is also much softer (although still hard; $\Gamma=1.44 \pm 0.05)$ and closer to those of PSRs J1023+0038 and J1227-4853 during the sub-luminous disk state, which are typically around $\Gamma \approx 1.6-1.8$ (e.g., de Martino et al. $2013 ; \mathrm{Li}$ et al. 2014; Bogdanov et al. 2015). These high intrinsic $N_{\mathrm{H}}$ and $\Gamma$ values lead the inferred nonthermal X-ray luminosity $(0.2-10 \mathrm{keV})$ to $L_{n \text {th }}=(1.75 \pm 0.05) \times 10^{33} \mathrm{erg} \mathrm{s}^{-1}$, which is also the highest among all the models. Despite the highest degree of freedom among the fits, the partially absorbed power-law fit has the best performance in terms of $\chi^{2}$ statistic, indicating that it is a better model for the Averagel spectrum statistically.

In the $84 \mathrm{ks}$ NuSTAR observation taken in 2016 May (about one year before the XMM-Newton observation), the photon index $\left(\Gamma_{\mathrm{nu}}=1.68_{-0.08}^{+0.09} ; \mathrm{S} 16\right)$ is much softer than any of the photon indices obtained in Averagel. The photon index deviation could be due to either (i) yearly spectral variability of 3FGL J0427.9 -6704 , or (ii) a high-energy exponential cut-off at $\gtrsim 10 \mathrm{keV}$. For 

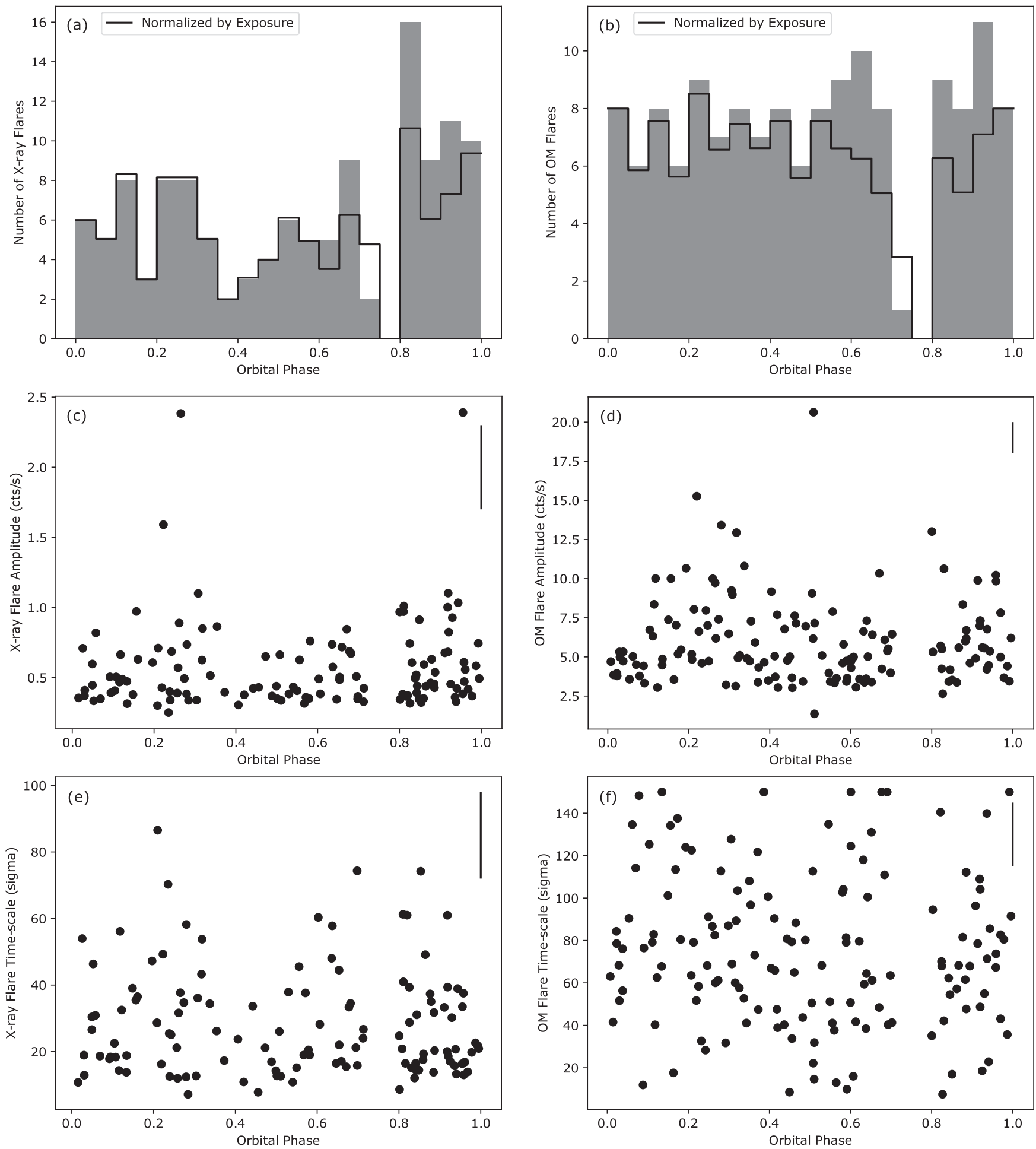

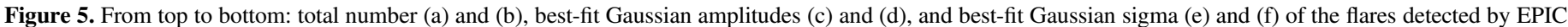

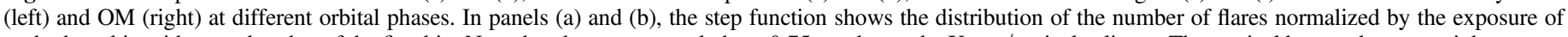

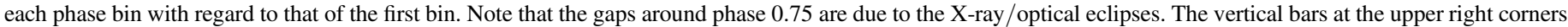
show the typical estimates of the uncertainties.

testing the latter case, we performed a joint spectral fitting of the Averagel and NuSTAR (obtained from S16) spectra with constant*phabs*pcfabs*cutoffpl, where cutoffpl can be written as $\frac{d N}{d E} \propto E^{-\Gamma} \exp \left(-\frac{E}{E_{\mathrm{c}}}\right)$. We fixed the pcfabs absorption component at the best-fit parameters obtained from Averagel for simplicity, and found that a power-law model with
$E_{\mathrm{c}} \approx 30 \mathrm{keV}$ can fit the joint XMM-Newton-NuSTAR spectrum very well, yielding a fitting statistic of $\chi_{\nu}^{2}=1.1$ (471 dof). However, we noticed that 3FGL J0427.9-6704 was about 40\% brighter in the NuSTAR observation (see the " $X+N$ " row in Table 1), strongly suggesting 3FGL J0427.9-6704 to be a longterm X-ray variable (e.g., the flare occurrence rate changes over a 


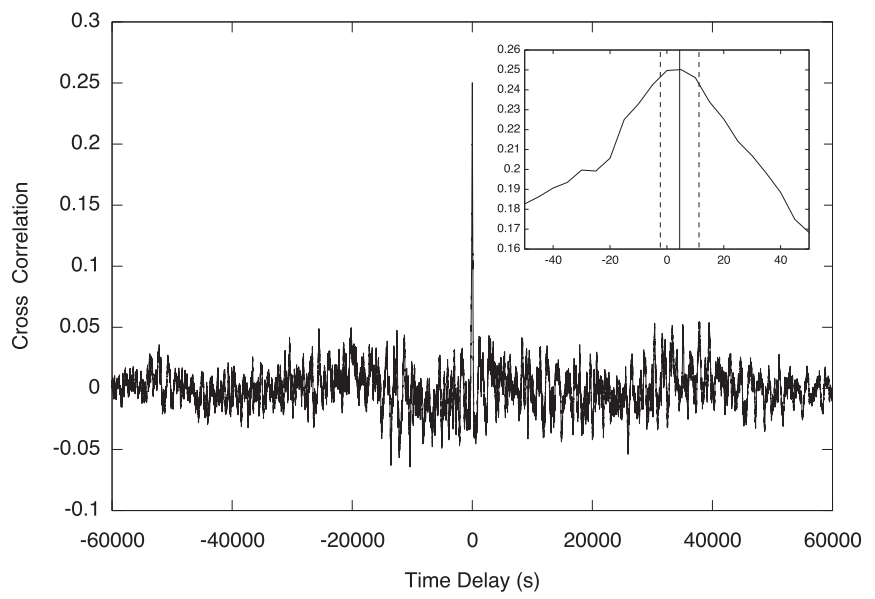

Figure 6. Cross-correlation function ( $5 \mathrm{~s}$ resolution) between the EPIC and the OM light curves computed by crosscor. The cross-correlations were normalized by dividing by $\sqrt{N_{1} N_{2}}$, where $N_{1}$ and $N_{2}$ are the numbers of bins in the two light curves, respectively. The three eclipse phases were excluded in the calculation. In the function, a positive time delay $\left(\Delta t_{d}\right)$ refers an optical/ $\mathrm{UV}$ emission delay with regard to the X-ray emission. No evidence of time delay is found in the analysis with $\Delta t_{d}=4.5 \pm 6.8 \mathrm{~s}$ (indicated by the vertical lines in the zoomed-in version in the inset box).

yearly timescale). Therefore, the observed high-energy cut-off may not be significant.

\subsubsection{Flux-resolved Spectra}

Like Averagel, the three flux-resolved spectra are all saddlelike. Nevertheless, their spectral shapes are slightly different from each other. From Lowl to Highl, the saddle-shaped feature becomes less obvious (Figure 8). With the same spectral models applied on Averagel, we characterized the spectral features of these groups. Except the simple absorbed power-law model, all the applied spectral models are equally good for the data statistically. ${ }^{7}$

Similar to Averagel, the inner disk radii inferred are all too small for a neutron star system. For the model composed of power and bbodyrad, the nonthermal X-ray emission still dominates the entire energy band. Although the photon index does not significantly change, the intrinsic $N_{\mathrm{H}}$ for the power-law component drops dramatically as the nonthermal X-ray luminosity increases (i.e., $N_{\mathrm{H}} / 10^{22} \mathrm{~cm}^{-2}$ : $9.2 \Rightarrow 6.3 \Rightarrow 4.1$ as $L_{n \text {th }}$ $/ 10^{33} \mathrm{erg} \mathrm{s}^{-1}: 0.8 \Rightarrow 2.9 \Rightarrow 5.4$ ). For the blackbody component, the temperature stays around $0.2-0.3 \mathrm{keV}$. The inferred emission size varies from group to group, but the changes are not significant as the uncertainties are high, e.g., all the sizes can be consistent with $R_{\mathrm{bb}} \approx 0.2 \mathrm{~km}$. Very similar results can be found in the nsa fits, with lower temperatures $\left(T_{\text {nsa }} \approx 0.1-0.2 \mathrm{keV}\right)$ and larger emission regions $\left(R_{\mathrm{nsa}} \approx 0.5-1.5 \mathrm{~km}\right)$.

For the partially absorbed power-law fits, the $N_{\mathrm{H}}$ decreasing trend aforementioned is still observed, however, with higher variability (i.e., $N_{\mathrm{H}} / 10^{22} \mathrm{~cm}^{-2}: 15 \Rightarrow 10 \Rightarrow 4.9$ as $L_{n \mathrm{th}} /$ $\left.10^{33} \mathrm{erg} \mathrm{s}^{-1}: 1.6 \Rightarrow 4.5 \Rightarrow 5.7\right)$. The photon index also becomes harder as the $\mathrm{X}$-ray luminosity goes up (i.e., $\Gamma: 1.8 \Rightarrow$ $1.6 \Rightarrow 1.2)$. Surprisingly, the coverage of the X-ray absorber does not change significantly among the flux-resolved groups ( $290 \%$ roughly). To further check whether this relation holds at lower luminosities, we extracted an extra set of spectra with MOS 1/2 data of $<0.1 \mathrm{cts} \mathrm{s}^{-1}$ (labeled as $C R<0.1$ in Table 1),

\footnotetext{
7 In Highl, the diskbb, bbody, and nsa temperatures were all fixed to the best-fit values obtained from Average. Otherwise, the fits do not converge.
}
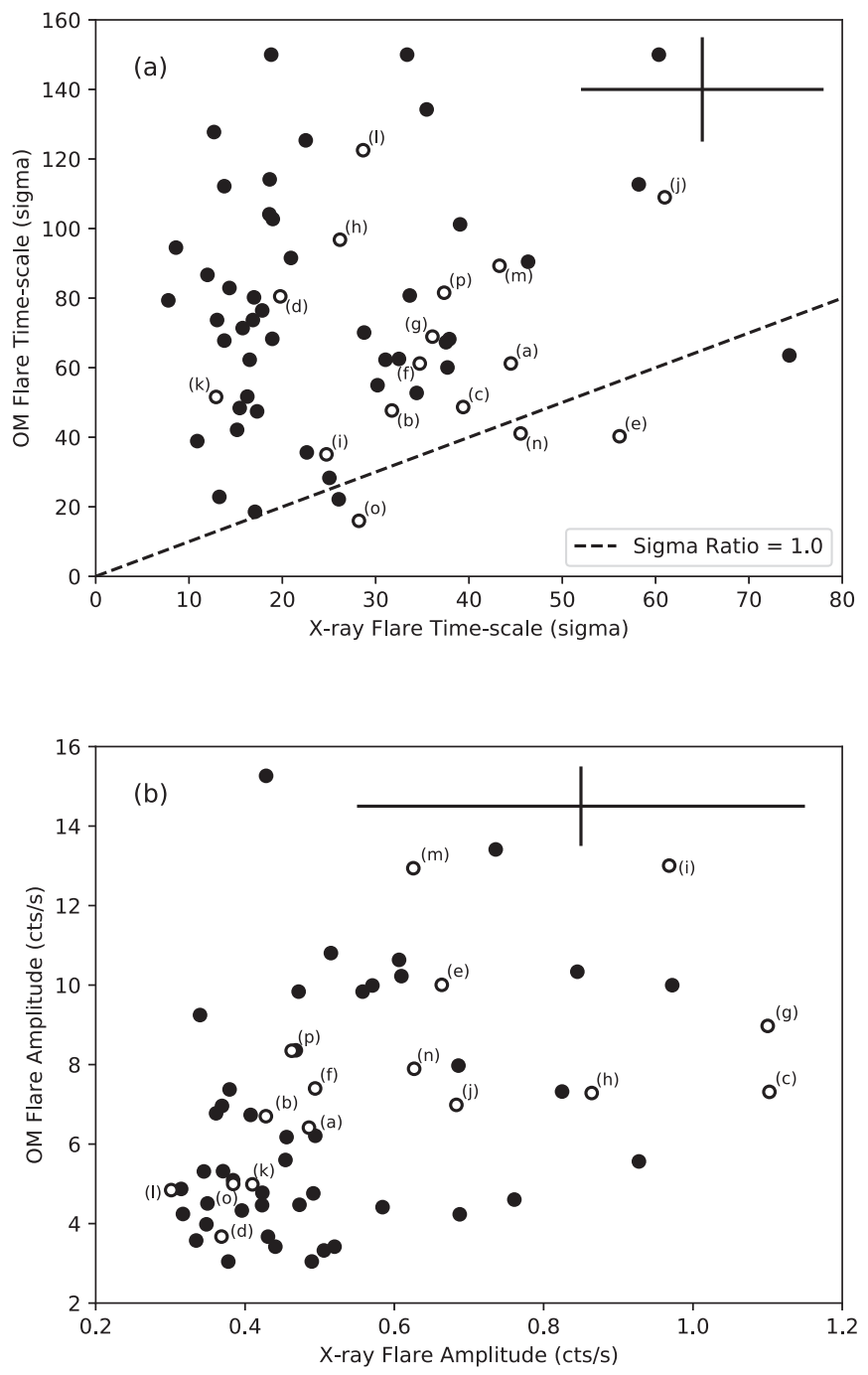

Figure 7. Comparisons between the flare characteristics observed in X-ray and optical/UV. Panel (a) refers to the flare timescale comparison, while panel (b) is for the flare amplitudes. In general, the flares last significantly longer in optical/UV than in X-rays (see the dashed line, which indicates the timescale ratio as 1). No clear relation can be found between the optical/UV and X-ray amplitudes. The 16 representative flares that are shown in Figure 4 are indicated by empty circles and labeled accordingly from (a) through (p). The crosses at the upper right corners show the typical estimates of the uncertainties.

which confirms the speculation with an even higher column density of $N_{\mathrm{H}} \approx 2 \times 10^{23} \mathrm{~cm}^{-2}$.

\subsubsection{Eclipse Spectra}

With the orbital solution presented in S16, we selected data collected in the three eclipse intervals for the following analysis. In addition, we excluded data within the first and last $200 \mathrm{~s}$ of the eclipses to avoid any residual emission in the eclipse ingress and egress (e.g., a possible mini-flare is seen in the last $\sim 100$ s of the third eclipse).

While there was no significant 3-79 $\mathrm{keV}$ emission detected by NuSTAR during the X-ray eclipses in 2016 (S16), we detected $\sim 50$ net counts in the pn and MOS 1/2 combined image. Given the low source counts and the high background noise $(\sim 50$ counts), we used $W$-statistic (a modified version of $C$-statistic; Cash 1979), which is able to handle Poisson-distributed 
Table 1

X-Ray Spectral Properties of 3FGL J0427.9-6704

\begin{tabular}{|c|c|c|c|c|c|c|c|c|c|c|c|}
\hline Dataset & Model $^{\mathrm{a}}$ & $\begin{array}{c}N_{\mathrm{H}} \\
\left(10^{22} \mathrm{~cm}^{-2}\right)\end{array}$ & $C_{1}^{\mathrm{b}}$ & $C_{2}{ }^{\mathrm{b}}$ & $\begin{array}{c}\text { Fraction } \\
(\%)\end{array}$ & $\Gamma$ & $\begin{array}{c}T \text { or } E_{\mathrm{c}} \\
(\mathrm{keV})\end{array}$ & $\begin{array}{l}\text { Radius } \\
\text { (m) }\end{array}$ & $\begin{array}{c}L_{n \text {th }} \\
\left(10^{33} \mathrm{erg} \mathrm{s}^{-1}\right)\end{array}$ & $\begin{array}{l}\frac{L_{n \text {th }}}{L_{\text {tot }}} \\
(\%)\end{array}$ & $\chi_{\nu}^{2}$ \\
\hline \multirow[t]{5}{*}{ Averagel } & pcfabs*pow & $10.9_{-0.5}^{+0.5}$ & $\cdots$ & $1.1_{-0.1}^{+0.1}$ & $96.5_{-1.2}^{+1.0}$ & $1.44_{-0.05}^{+0.05}$ & $\cdots$ & $\cdots$ & $1.75_{-0.05}^{+0.05}$ & $\cdots$ & $197.7 / 187$ \\
\hline & phabs*pow & $3.8_{-1.0}^{+1.0}$ & $\ldots$ & $1.0_{-0.1}^{+0.1}$ & $\ldots$ & $0.48_{-0.22}^{+0.22}$ & $\ldots$ & $\ldots$ & $1.02_{-0.07}^{+0.08}$ & $\ldots$ & $450.3 / 188$ \\
\hline & diskbb+phabs*pow & $7.7_{-1.6}^{+2.0}$ & $\cdots$ & $1.1_{-0.1}^{+0.1}$ & $\cdots$ & $1.07_{-0.26}^{+0.28}$ & $0.52_{-0.12}^{+0.23}$ & $84_{-41}^{+55}$ & $1.32_{-0.17}^{+0.29}$ & 98 & $203.3 / 186$ \\
\hline & bbody+phabs*pow & $6.5_{-1.2}^{+1.5}$ & $\cdots$ & $1.0_{-0.1}^{+0.1}$ & $\cdots$ & $0.91_{-0.23}^{+0.26}$ & $0.27_{-0.04}^{+0.05}$ & $154_{-46}^{+56}$ & $1.20_{-0.12}^{+0.19}$ & 99 & $217.3 / 186$ \\
\hline & nsa+phabs*pow ${ }^{\mathrm{c}}$ & $6.8_{-1.3}^{+1.4}$ & $\cdots$ & $1.0_{-0.1}^{+0.1}$ & $\cdots$ & $0.95_{-0.24}^{+0.25}$ & $0.18_{-0.04}^{+0.05}$ & $507_{-195}^{+328}$ & $1.22_{-0.13}^{+0.19}$ & 99 & $211.0 / 186$ \\
\hline \multirow[t]{5}{*}{ Low1 } & pcfabs*pow & $15.4_{-0.8}^{+0.9}$ & $\cdots$ & $1.1_{-0.1}^{+0.1}$ & $97.9_{-1.2}^{+0.8}$ & $1.75_{-0.05}^{+0.05}$ & $\cdots$ & $\cdots$ & $1.61_{-0.07}^{+0.07}$ & $\cdots$ & $114.4 / 95$ \\
\hline & phabs*pow & $6.9_{-2.3}^{+2.7}$ & $\cdots$ & $1.1_{-0.1}^{+0.1}$ & $\ldots$ & $0.75_{-0.39}^{+0.43}$ & $\ldots$ & $\ldots$ & $0.71_{-0.10}^{+0.18}$ & $\cdots$ & $267.4 / 96$ \\
\hline & diskbb+phabs*pow & $10.4_{-2.5}^{+2.9}$ & $\ldots$ & $1.1_{-0.1}^{+0.1}$ & $\cdots$ & $1.17_{-0.39}^{+0.43}$ & $0.42_{-0.08}^{+0.13}$ & $113_{-47}^{+64}$ & $0.90_{-0.18}^{+0.40}$ & 98 & $113.2 / 94$ \\
\hline & bbody+phabs*pow & $9.2_{-2.3}^{+2.7}$ & $\ldots$ & $1.1_{-0.1}^{+0.1}$ & $\ldots$ & $1.04_{-0.38}^{+0.42}$ & $0.24_{-0.03}^{+0.04}$ & $170_{-49}^{+61}$ & $0.82_{-0.14}^{+0.29}$ & 98 & $118.2 / 94$ \\
\hline & nsa+phabs*pow & $9.8_{-2.4}^{+2.7}$ & $\cdots$ & $1.1_{-0.1}^{+0.1}$ & $\cdots$ & $1.11_{-0.38}^{+0.42}$ & $0.16_{-0.03}^{+0.04}$ & $581_{-226}^{+366}$ & $0.86_{-0.16}^{+0.33}$ & 98 & $115.0 / 94$ \\
\hline \multirow[t]{5}{*}{ Medium1 } & pcfabs*pow & $10.4_{-0.7}^{+0.8}$ & $\cdots$ & $1.0_{-0.1}^{+0.1}$ & $98.0_{-1.8}^{+0.9}$ & $1.60_{-0.08}^{+0.07}$ & $\cdots$ & $\cdots$ & $4.53_{-0.25}^{+0.25}$ & $\cdots$ & $51.0 / 48$ \\
\hline & phabs*pow & $5.1_{-1.6}^{+1.8}$ & $\ldots$ & $1.0_{-0.1}^{+0.1}$ & $\ldots$ & $0.77_{-0.39}^{+0.41}$ & $\ldots$ & $\ldots$ & $2.59_{-0.34}^{+0.55}$ & $\ldots$ & $112.7 / 49$ \\
\hline & diskbb+phabs $*$ pow & $6.8_{-1.8}^{+2.4}$ & $\ldots$ & $1.0_{-0.1}^{+0.1}$ & $\ldots$ & $1.05_{-0.39}^{+0.45}$ & $0.34_{-0.09}^{+0.23}$ & $269_{-178}^{+301}$ & $2.98_{-0.49}^{+1.08}$ & 99 & $49.5 / 47$ \\
\hline & bbody+phabs*pow & $6.3_{-1.7}^{+2.1}$ & $\cdots$ & $1.0_{-0.1}^{+0.1}$ & $\cdots$ & $0.98_{-0.38}^{+0.42}$ & $0.21_{-0.04}^{+0.07}$ & $374_{-182}^{+266}$ & $2.86_{-0.43}^{+0.84}$ & 99 & $51.9 / 47$ \\
\hline & nsa+phabs*pow & $6.6_{-1.7}^{+2.1}$ & $\ldots$ & $1.0_{-0.1}^{+0.1}$ & $\ldots$ & $1.02_{-0.39}^{+0.42}$ & $0.12_{-0.04}^{+0.06}$ & $1550_{-943}^{+2162}$ & $2.93_{-0.46}^{+0.92}$ & 99 & $50.1 / 47$ \\
\hline \multirow[t]{5}{*}{ Highl } & pcfabs*pow & $4.9_{-0.5}^{+0.6}$ & $\cdots$ & $1.0_{-0.1}^{+0.1}$ & $95.7_{-3.4}^{+2.2}$ & $1.22_{-0.13}^{+0.12}$ & $\cdots$ & $\cdots$ & $5.69_{-0.36}^{+0.36}$ & $\cdots$ & $21.9 / 33$ \\
\hline & phabs*pow & $2.7_{-0.8}^{+1.1}$ & $\ldots$ & $1.0_{-0.1}^{+0.1}$ & $\ldots$ & $0.86_{-0.31}^{+0.34}$ & $\ldots$ & $\ldots$ & $4.63_{-0.59}^{+0.80}$ & $\cdots$ & $46.9 / 34$ \\
\hline & diskbb+phabs*pow & $4.4_{-1.2}^{+1.6}$ & $\ldots$ & $1.0_{-0.1}^{+0.1}$ & $\ldots$ & $1.20_{-0.36}^{+0.40}$ & $(0.52)^{\mathrm{d}}$ & $144_{-26}^{+22}$ & $5.55_{-0.97}^{+2.00}$ & 99 & $22.9 / 33$ \\
\hline & bbody+phabs*pow & $4.1_{-1.1}^{+1.5}$ & $\cdots$ & $1.0_{-0.1}^{+0.1}$ & $\cdots$ & $1.16_{-0.34}^{+0.38}$ & $(0.27)^{\mathrm{d}}$ & $264_{-49}^{+41}$ & $5.38_{-0.87}^{+1.66}$ & 99 & $23.2 / 33$ \\
\hline & nsa+phabs*pow & $4.1_{-1.1}^{+1.5}$ & $\ldots$ & $1.0_{-0.1}^{+0.1}$ & $\cdots$ & $1.15_{-0.34}^{+0.39}$ & $(0.18)^{\mathrm{d}}$ & $855_{-158}^{+134}$ & $5.37_{-0.87}^{+1.67}$ & 99 & $23.4 / 33$ \\
\hline \multirow[t]{5}{*}{ Average2 } & pcfabs*pow & $10.4_{-0.4}^{+0.4}$ & $1.0_{-0.1}^{+0.1}$ & $1.0_{-0.1}^{+0.1}$ & $96.5_{-0.9}^{+0.8}$ & $1.43_{-0.04}^{+0.04}$ & $\cdots$ & $\cdots$ & $1.74_{-0.04}^{+0.04}$ & $\ldots$ & $320.0 / 300$ \\
\hline & phabs*pow & $4.3_{-0.7}^{+0.8}$ & $1.0_{-0.1}^{+0.1}$ & $1.0_{-0.1}^{+0.1}$ & $\ldots$ & $0.69_{-0.16}^{+0.16}$ & $\ldots$ & $\ldots$ & $1.03_{-0.07}^{+0.08}$ & $\cdots$ & $750.0 / 301$ \\
\hline & diskbb+phabs*pow & $7.9_{-1.2}^{+1.4}$ & $1.0_{-0.1}^{+0.1}$ & $1.0_{-0.1}^{+0.1}$ & $\cdots$ & $1.18_{-0.18}^{+0.20}$ & $0.54_{-0.09}^{+0.15}$ & $78_{-28}^{+34}$ & $1.39_{-0.17}^{+0.25}$ & 98 & $327.9 / 299$ \\
\hline & bbody+phabs*pow & $6.7_{-0.9}^{+1.1}$ & $1.0_{-0.1}^{+0.1}$ & $1.0_{-0.1}^{+0.1}$ & $\ldots$ & $1.03_{-0.17}^{+0.18}$ & $0.27_{-0.03}^{+0.03}$ & $156_{-31}^{+36}$ & $1.25_{-0.12}^{+0.16}$ & 99 & $347.6 / 299$ \\
\hline & nsa+phabs*pow & $7.0_{-1.0}^{+1.0}$ & $1.0_{-0.1}^{+0.1}$ & $1.0_{-0.1}^{+0.1}$ & $\cdots$ & $1.07_{-0.17}^{+0.18}$ & $0.18_{-0.03}^{+0.03}$ & $476_{-126}^{+186}$ & $1.28_{-0.13}^{+0.17}$ & 99 & $338.5 / 299$ \\
\hline \multirow[t]{5}{*}{ Low2 } & pcfabs*pow & $15.4_{-0.7}^{+0.7}$ & $1.0_{-0.1}^{+0.1}$ & $1.1_{-0.1}^{+0.1}$ & $97.7_{-0.9}^{+0.6}$ & $1.74_{-0.04}^{+0.04}$ & $\cdots$ & $\cdots$ & $1.58_{-0.06}^{+0.06}$ & $\cdots$ & $171.4 / 154$ \\
\hline & phabs*pow & $7.2_{-1.8}^{+2.0}$ & $1.1_{-0.1}^{+0.1}$ & $1.1_{-0.1}^{+0.1}$ & $\ldots$ & $0.85_{-0.29}^{+0.31}$ & $\ldots$ & $\ldots$ & $0.69_{-0.10}^{+0.15}$ & $\ldots$ & $455.5 / 155$ \\
\hline & diskbb+phabs*pow & $10.6_{-2.0}^{+2.2}$ & $1.0_{-0.1}^{+0.1}$ & $1.1_{-0.1}^{+0.1}$ & $\cdots$ & $1.24_{-0.29}^{+0.32}$ & $0.42_{-0.06}^{+0.08}$ & $112_{-34}^{+42}$ & $0.92_{-0.17}^{+0.31}$ & 98 & $168.3 / 153$ \\
\hline & bbody+phabs*pow & $9.4_{-1.8}^{+2.1}$ & $1.0_{-0.1}^{+0.1}$ & $1.1_{-0.1}^{+0.1}$ & $\cdots$ & $1.12_{-0.28}^{+0.31}$ & $0.24_{-0.02}^{+0.03}$ & $175_{-36}^{+42}$ & $0.83_{-0.14}^{+0.23}$ & 98 & $175.9 / 153$ \\
\hline & nsa+phabs*pow & $10.0_{-1.9}^{+2.1}$ & $1.0_{-0.1}^{+0.1}$ & $1.1_{-0.1}^{+0.1}$ & $\cdots$ & $1.18_{-0.29}^{+0.30}$ & $0.16_{-0.02}^{+0.03}$ & $579_{-161}^{+238}$ & $0.87_{-0.15}^{+0.26}$ & 98 & $170.7 / 153$ \\
\hline \multirow[t]{5}{*}{ Medium2 } & pcfabs*pow & $10.3_{-0.6}^{+0.6}$ & $1.0_{-0.1}^{+0.1}$ & $1.0_{-0.1}^{+0.1}$ & $98.2_{-1.0}^{+0.7}$ & $1.58_{-0.06}^{+0.06}$ & $\cdots$ & $\cdots$ & $4.62_{-0.20}^{+0.20}$ & $\cdots$ & $73.5 / 79$ \\
\hline & phabs*pow & $6.2_{-1.3}^{+1.5}$ & $1.0_{-0.1}^{+0.1}$ & $1.0_{-0.1}^{+0.1}$ & $\ldots$ & $1.07_{-0.28}^{+0.30}$ & $\ldots$ & $\ldots$ & $2.92_{-0.44}^{+0.71}$ & $\ldots$ & $181.3 / 80$ \\
\hline & diskbb+phabs*pow & $8.2_{-1.6}^{+2.1}$ & $1.0_{-0.1}^{+0.1}$ & $1.0_{-0.1}^{+0.1}$ & $\cdots$ & $1.32_{-0.30}^{+0.33}$ & $0.43_{-0.12}^{+0.28}$ & $153_{-94}^{+144}$ & $3.54_{-0.71}^{+1.41}$ & 99 & $75.9 / 78$ \\
\hline & bbody+phabs*pow & $7.4_{-1.4}^{+1.7}$ & $1.0_{-0.1}^{+0.1}$ & $1.0_{-0.1}^{+0.1}$ & $\cdots$ & $1.23_{-0.29}^{+0.31}$ & $0.23_{-0.04}^{+0.07}$ & $267_{-107}^{+140}$ & $3.27_{-0.58}^{+1.03}$ & 99 & $80.8 / 78$ \\
\hline & nsa+phabs*pow & $7.7_{-1.5}^{+1.7}$ & $1.0_{-0.1}^{+0.1}$ & $1.0_{-0.1}^{+0.1}$ & $\cdots$ & $1.26_{-0.29}^{+0.31}$ & $0.15_{-0.04}^{+0.06}$ & $939_{-469}^{+915}$ & $3.36_{-0.62}^{+1.10}$ & 99 & $78.0 / 78$ \\
\hline \multirow[t]{5}{*}{ High2 } & pcfabs*pow & $3.7_{-0.3}^{+0.4}$ & $1.1_{-0.1}^{+0.1}$ & $1.1_{-0.1}^{+0.1}$ & $95.4_{-3.0}^{+1.9}$ & $1.02_{-0.10}^{+0.10}$ & $\cdots$ & $\cdots$ & $4.58_{-0.23}^{+0.23}$ & $\cdots$ & $40.5 / 56$ \\
\hline & phabs*pow & $2.3_{-0.6}^{+0.7}$ & $1.1_{-0.1}^{+0.1}$ & $1.1_{-0.1}^{+0.1}$ & $\ldots$ & $0.78_{-0.24}^{+0.25}$ & $\ldots$ & $\ldots$ & $4.14_{-0.40}^{+0.47}$ & $\cdots$ & $69.1 / 57$ \\
\hline & diskbb+phabs*pow & $3.4_{-0.8}^{+0.9}$ & $1.1_{-0.1}^{+0.1}$ & $1.1_{-0.1}^{+0.1}$ & $\ldots$ & $1.00_{-0.25}^{+0.27}$ & $(0.54)^{\mathrm{d}}$ & $106_{-18}^{+15}$ & $4.50_{-0.51}^{+0.72}$ & 99 & $41.9 / 56$ \\
\hline & bbody+phabs*pow & $5.7_{-1.9}^{+7.9}$ & $1.1_{-0.1}^{+0.1}$ & $1.1_{-0.1}^{+0.1}$ & $\cdots$ & $1.10_{-0.40}^{+2.68}$ & $1.25_{-0.98}^{+1.85}$ & $40_{-19}^{+143}$ & $4.36_{-1.88}^{+2.46}$ & 90 & $37.9 / 55$ \\
\hline & nsa+phabs*pow & $3.2_{-0.7}^{+0.9}$ & $1.1_{-0.1}^{+0.1}$ & $1.1_{-0.1}^{+0.1}$ & $\ldots$ & $0.98_{-0.25}^{+0.27}$ & $(0.18)^{\mathrm{d}}$ & $638_{-108}^{+92}$ & $4.46_{-0.49}^{+0.68}$ & 99 & $42.0 / 56$ \\
\hline Eclipse $^{\mathrm{e}}$ & pow & $\cdots$ & $(1.0)$ & $(1.0)$ & $\cdots$ & $1.90_{-0.43}^{+0.45}$ & $\cdots$ & $\cdots$ & $0.03_{-0.01}^{+0.01}$ & $\cdots$ & $\cdots$ \\
\hline$X+N^{\mathrm{f}}$ & pcfabs*cutoffpl & $(10.9)$ & $1.4_{-0.1}^{+0.1}$ & $1.4_{-0.1}^{+0.1}$ & $(96.5)$ & $1.43_{-0.07}^{+0.07}$ & $31_{-7}^{+13}$ & $\cdots$ & $1.76_{-0.07}^{+0.07}$ & $\cdots$ & $502.8 / 471$ \\
\hline$C R<0.1$ & pcfabs*pow & $20.8_{-2.4}^{+3.0}$ & $\ldots$ & $1.1_{-0.2}^{+0.2}$ & $98.2_{-4.2}^{+1.4}$ & $1.75_{-0.13}^{+0.13}$ & $\ldots$ & $\cdots$ & $0.91_{-0.11}^{+0.11}$ & $\ldots$ & $17.0 / 18$ \\
\hline
\end{tabular}

Notes.

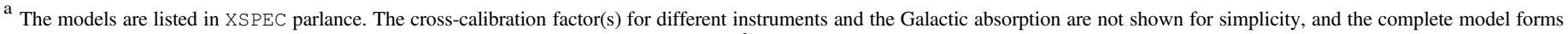

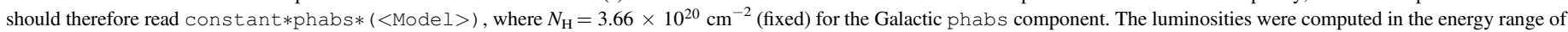
$0.2-10 \mathrm{keV}$ with $d=2.3 \mathrm{kpc}$.

${ }^{\mathrm{b}}$ Except for $X+N, C_{1}$ (or $C_{2}$ ) is the cross-calibration factor for MOS 1 (MOS 2) with regard to pn. (MOS 1 or pn).

${ }^{\mathrm{c}}$ For nsa, a non-magnetized (i.e., $B<10^{9} \mathrm{G}$ ) neutron star of $M_{\mathrm{NS}}=1.4 M_{\odot}$ and $R_{\mathrm{NS}}=10 \mathrm{~km}$ is assumed.

$\mathrm{d}$ The temperatures that could not be well converged were fixed to the values obtained from Average/Average 2.

e As the data quality is low, the cross-calibration factors were both fixed to 1 . $C$ statistic was also applied $(C=124.0$ with 132 dof $)$.

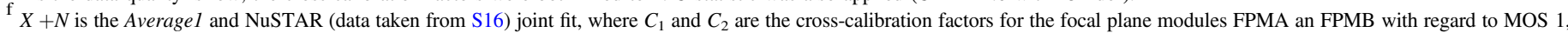
respectively. The intrinsic $N_{\mathrm{H}}$, the cross-calibration factor for MOS 2, and the Fraction parameter for pcfabs were all fixed to the values obtained from Averagel for simplicity. 
Flux-Resolved Spectra of 3FGL J0427.9-6704
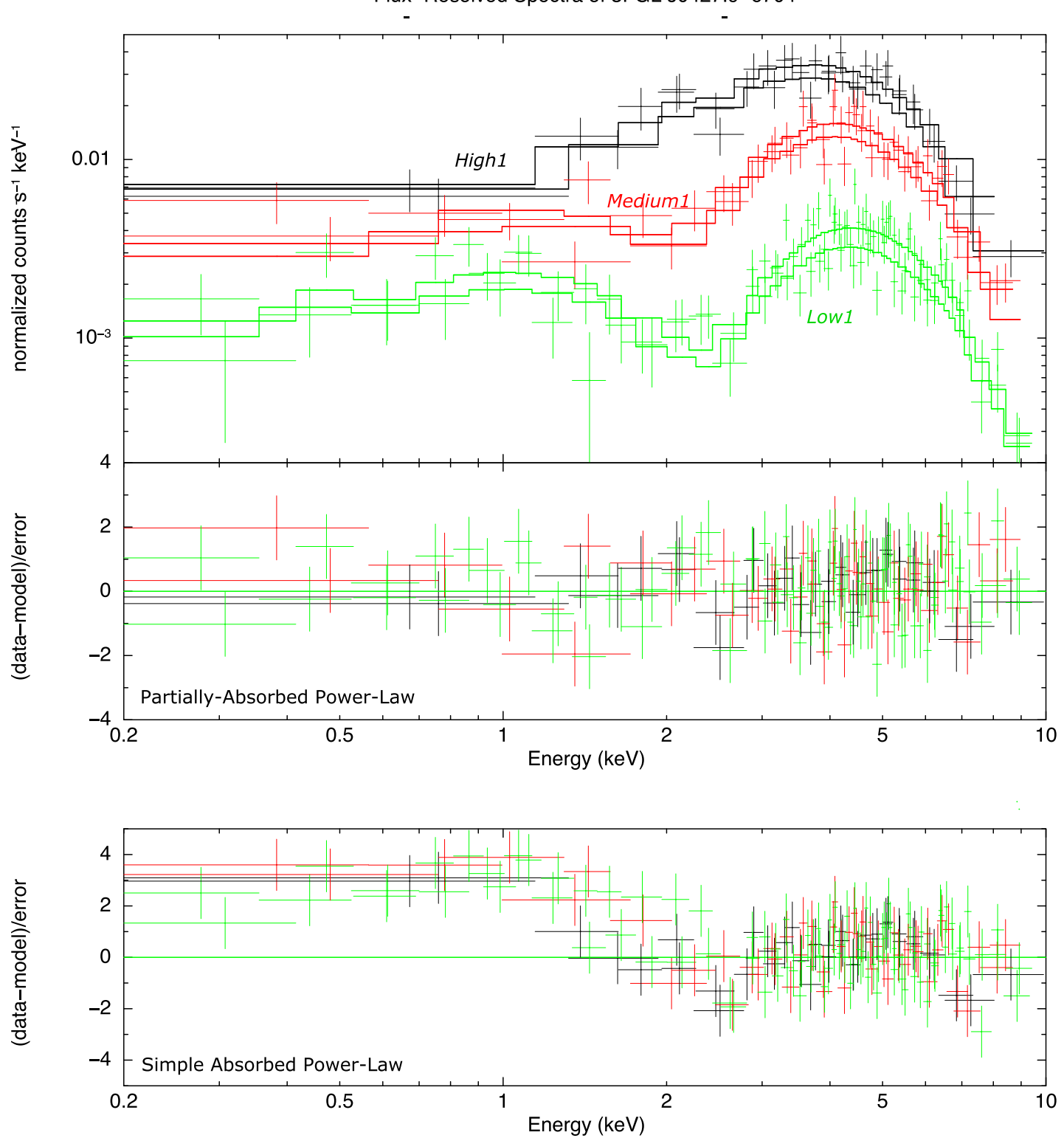

Figure 8. Upper: XMM-Newton (MOS 1/2) phase-resolved spectra of 3FGL J0427.9-6704 with the best-fit partially absorbed power-law models (see Table 1 for the best-fit parameters). From bright to faint, the spectra are classified into High1 ( $>0.6$ counts s ${ }^{-1}$; black), Medium1 (0.3-0.6 counts s ${ }^{-1}$; red), and Low1 ( $<$ 0.3 counts $\mathrm{s}^{-1}$; green). The detailed scheme of classification can be found in Section 3.2. Lower: a residual plot with respect to the best-fit simple absorbed power-law models.

background spectra. We binned the spectra using grppha to at least one count per bin as suggested in the XSPEC manual. A simple absorbed power law with the hydrogen column density fixed at the Galactic value of $N_{\mathrm{H}}=3.66 \times 10^{20} \mathrm{~cm}^{-2}$ was used as a phenomenological model. No intrinsic absorption is assumed because (i) the limited data quality does not allow a good probe for an intrinsic absorption, and (ii) the X-ray emission observed during the eclipse is likely already scattered away from the absorbing material in the disk. In addition, no cross-calibration correction was applied on the spectral model (i.e., the crosscalibration factors were fixed to 1) because of the low $\mathrm{S} / \mathrm{N}$. The best-fit photon index was $\Gamma=1.9_{-0.4}^{+0.5}$, with an inferred luminosity of $L_{x} \approx 3 \times 10^{31} \mathrm{erg} \mathrm{s}^{-1}(0.2-10 \mathrm{keV})$, which is about $2 \%$ of the X-ray luminosity in Averagel.

These relatively soft X-rays are possibly scattered from the atmosphere of the companion, or in an extended accretion disk corona (ADC; White \& Holt 1982). In the former case, the scattered emission is strong only during the eclipse when the pulsar is behind the scattering medium. The ADC emission, by contrast, is weakest during the eclipse, and can be observed in all other phases. To examine this possible ADC component, we added the eclipse emission spectrum (fixed at the best-fit parameters) to the partially absorbed power-law model, and fit the composite model to the flux-resolved spectra (Table 2). In general, the fits are not improved. For the best-fit parameters, while there is almost no change on Highl, the power-law components of Lowl and Mediuml are significantly harder than the previous ones. In particular the pcfabs component of Lowl is no longer required (fraction $=1$ ) as the eclipse emission cancels out the soft X-ray excess. However, we argue that this is likely a coincidence. Although an extended ADC is possibly observable during the eclipse, a large fraction of it is still occult. 
Table 2

pcfabs Fittings of 3FGL J0427.9-6704 (Eclipse Emission Subtracted)

\begin{tabular}{|c|c|c|c|c|c|c|c|}
\hline Dataset & Model & $\begin{array}{c}N_{\mathrm{H}} \\
\left(10^{22} \mathrm{~cm}^{-2}\right)\end{array}$ & $C_{2}$ & $\begin{array}{l}\text { Fraction } \\
(\%)\end{array}$ & $\Gamma$ & $\left(10^{33} \mathrm{erg} \mathrm{s}^{L_{n t h}}\right)$ & $\chi_{\nu}^{2}$ \\
\hline Low1 & pcfabs*pow & $11.9_{-2.6}^{+0.8}$ & $1.1_{-0.1}^{+0.1}$ & 100 & $1.28_{-0.11}^{+0.10}$ & $0.99_{-0.05}^{+0.53}$ & $117.0 / 95$ \\
\hline Medium 1 & pcfabs*pow & $9.6_{-0.7}^{+0.8}$ & $1.0_{-0.1}^{+0.1}$ & $98.3_{-1.6}^{+0.9}$ & $1.42_{-0.10}^{+0.10}$ & $3.86_{-0.22}^{+0.22}$ & $50.0 / 48$ \\
\hline High1 & pcfabs*pow & $4.8_{-0.5}^{+0.6}$ & $1.0_{-0.1}^{+0.1}$ & $96.6_{-3.0}^{+1.7}$ & $1.21_{-0.14}^{+0.13}$ & $5.63_{-0.36}^{+0.36}$ & $22.2 / 33$ \\
\hline
\end{tabular}

Note.

${ }^{\text {a }}$ See the caption of Table 1 for details.

Much brighter ADC emission should therefore be observed in Lowl unless the ADC is extremely extended. For LMXBs, the $\mathrm{ADC}$ can extend up to $1 R_{\odot}$ in the radial direction (Church $\&$ Bałucińska-Church 2004), which just slightly exceeds the companion of 3FGL J0427.9-6704 $\left(R_{2}=0.83 R_{\odot} ; \mathrm{S} 16\right)$, not to mention that such an extended ADC requires a powerful central engine of $L_{X} \sim 10^{38} \mathrm{erg} \mathrm{s}^{-1}$ (Church \& Bałucińska-Church 2004) that is almost 1000 times higher than that of 3FGL J0427.9 -6704. Thus, the consistency between the soft X-ray excess in Low1 and the eclipse emission actually disfavors the ADC scattering scenario. As a result, we assumed the X-ray scattering off the companion's atmosphere as the origin of the eclipse emission, which would only bring a minor effect to our spectral analysis.

\section{Radio Continuum Results}

Figure 9 shows the 5.5 and $9.0 \mathrm{GHz}$ light curves of 3FGL J0427.9-6704, overlaid on the simultaneous X-ray light curve for the same time period. As discussed above, the radio data are binned at time intervals of $10 \mathrm{~min}(600 \mathrm{~s})$.

The main results from the radio data are as follows. First, 3FGL J0427.9-6704 is well-detected in both frequency bands at all times, with a mean out-of-eclipse flux density of $290 \pm 7$ and $300 \pm 6 \mu \mathrm{Jy}$ at 5.5 and $9.0 \mathrm{GHz}$, respectively. For a power-law spectrum with flux density $S_{\nu} \propto \nu^{\alpha}$, these values imply a mean $\alpha=0.07 \pm 0.07$. Second, there is no evidence that the radio emission is eclipsed, with mean in-eclipse flux densities of $295 \pm 21$ and $334 \pm 18 \mu \mathrm{Jy}$ at 5.5 and $9.0 \mathrm{GHz}$. Hence the radio emission must primarily arise on size scales larger than the projected secondary $\left(\gtrsim 3.5 \times 10^{10} \mathrm{~cm}\right.$, using the parameters from S16).

Next, while the radio continuum flux density is timevariable, no obvious flares can be discerned, quite unlike the X-ray and optical/UV light curves (Figure 9). Given that the $\mathrm{X}$-ray flares typically last for $10-40 \mathrm{~s}$ and the radio light curve is binned on a timescale of $600 \mathrm{~s}$, the lack of obvious flares in the radio light curves is perhaps not surprising. However, we do find some evidence that higher X-ray emission is associated with higher radio emission. If we consider the radio emission in the Low1, Medium1, and High1 X-ray categories described earlier, the Lowl and Mediuml categories have mean radio flux densities consistent with the full data set, while the Highl $\mathrm{X}$-ray category is associated with a $5.5 \mathrm{GHz}$ flux density of $427 \pm 36 \mu \mathrm{Jy}$ (brighter at $3.8 \sigma$ ) and a marginally steeper radio spectrum of $\alpha=-0.68 \pm 0.39$.

Table 3 shows the flux densities as well as the spectral indices of these subsets of the radio data.

\subsection{Older Radio Data}

Here we briefly discuss the results from the 2016 August radio continuum observations of 3FGL J0427.9-6704. These were not taken simultaneously with any X-ray observations.

We find flux densities of $303 \pm 9$ and $337 \pm 8 \mu \mathrm{Jy}$ at 5.5 and $9.0 \mathrm{GHz}$, respectively, giving a spectral index of $\alpha=0.21 \pm 0.08$. These values are entirely consistent with those measured in 2017 May, and hence show that at least over the approximate nine month separation of these epochs that the radio behavior of the binary is stable.

\section{Discussion}

Individual X-ray flaring events are not uncommon in redback and black widow systems, e.g., PSR J1048+2339 (Cho et al. 2018; Yap et al. 2019), 3FGL J0838.8-2829 (Halpern et al. 2017), and PSR J1311-3430 (Romani 2012; Romani et al. 2015; An et al. 2017), but this paper is the first to show evidence for a system with a fully flare-dominated accretion mode. Perhaps the closest comparison is the few flare-dominated epochs of PSR J1023+0038 in its subluminous disk state (Tendulkar et al. 2014; Li et al. 2014; Bogdanov et al. 2015; Papitto et al. 2019), though these have typically been short-lived, and make up only a small fraction of the observed modes in the current accretion state of PSR J1023 +0038 . By contrast, 3FGL J0427.9-6704 maintained this flare-dominated state during the entirety of our $\sim 70 \mathrm{ks}$ observations in 2017 May and, despite the lower sensitivity of earlier X-ray observations, appears to have been in a similar state in 2016 May and likely much earlier (see S16).

\subsection{The Concurrent Optical/UV Flares}

Given the large dispersion in the flare amplitudes between X-rays and optical/UV (Figure 7), the emission mechanisms of the two bands are probably different. Another intriguing property is that the optical/UV flares are generally longer than the X-ray flares (Figure 7), implying that that the optical/UV flares are emitted from the more outer region (e.g., the accretion disk).

Perhaps the simplest model is that the optical/UV flares are the reprocessed emission from the X-rays, for example, due to "reflection" of the accretion disk. Considering the light-travel time, there should be a minimum time delay in the optical/UV light curve of at least $\sim 2.3 \mathrm{~s}$ to the outer disk. Our analysis found an insignificant delay of $4.5 \pm 6.8 \mathrm{~s}$, which is consistent with (but does not constrain) this expected delay. We also note that several optical/UV flares appear to have started earlier than their X-ray counterpart (see Figure 4(h) as the most prominent case), which would not be consistent with this 

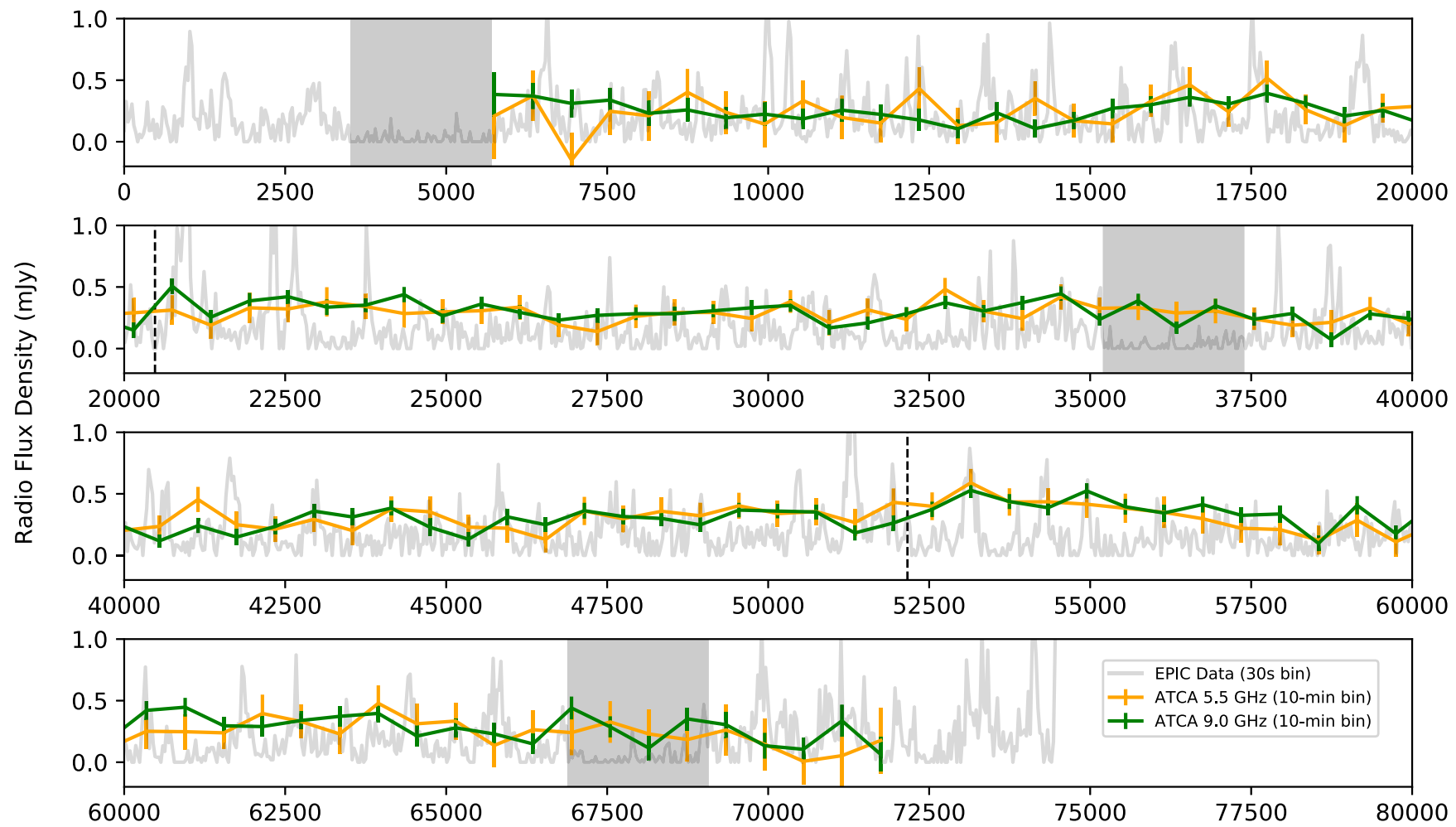

Barycentric Time since BJD (TDB) $=2457876.20508$ (seconds)

Figure 9. Similar light curve to Figure 2, but with the ATCA 5.5 and $9.0 \mathrm{GHz}$ radio continuum data. The radio continuum data show modest variability but no clear flares, and there is no evidence that the radio emission is eclipsed.

Table 3

ATCA Radio Properties of 3FGL J0427.9-6704 in 2017 May

\begin{tabular}{lccc}
\hline \hline Dataset & $\begin{array}{c}5.5 \mathrm{GHz} \\
(\mu \mathrm{Jy})\end{array}$ & $\begin{array}{c}9.0 \mathrm{GHz} \\
(\mu \mathrm{Jy})\end{array}$ & $\begin{array}{c}\text { Spectral Index } \\
(\alpha)\end{array}$ \\
\hline In eclipse & $295 \pm 21$ & $334 \pm 18$ & $0.25 \pm 0.22$ \\
Out of eclipse & $290 \pm 7$ & $300 \pm 6$ & $0.07 \pm 0.07$ \\
Low1 & $281 \pm 8$ & $302 \pm 6$ & $0.15 \pm 0.10$ \\
Medium1 & $307 \pm 20$ & $268 \pm 16$ & $-0.28 \pm 0.22$ \\
High1 & $427 \pm 36$ & $305 \pm 27$ & $-0.68 \pm 0.39$ \\
\hline
\end{tabular}

simple model. Coordinated multi-wavelength observations by an X-ray timing instrument such as NICER and ground-based telescopes capable of fast photometry could clarify this in the future.

\subsection{Intrinsic X-Ray Variability or Rapidly Varying $\mathrm{N}_{H}$ ?}

Our flux-resolved X-ray spectral analysis showed an enhancement in $N_{\mathrm{H}}$ when the X-ray flux becomes faint. This is reminiscent of variable absorption, instead of an intrinsic flux change, resulting in the strong variability observed. The idea has also been used to explain the X-ray variability of the edgeon LMXB, 47 Tuc-X5, observed by Chandra (Heinke et al. 2003; Bogdanov et al. 2016). The variable $N_{\mathrm{H}}$ could be due to a precessing accretion disk that varies the obscuring gas in the line of sight. Optical emission from the outer disk region would then have a better chance than the X-rays from the inner part to be seen through a cloud gap. This naturally explains the nonsymmetric relation between the X-ray and optical flares as well as the shorter flaring timescales in X-rays.
An immediate objection is that the spectral fits find significant variations of the unabsorbed X-ray flux and the photon index. Although this might be attributed to imperfect correction for the absorption if each spectral data set still contains too wide a range of fluxes, it is questionable whether the effect is sufficient to remove the variations. Additionally, the X-ray and optical/UV flares would be at least weakly correlated if they both originate from obscuration. However, we find no correlation in Figure 7. The contradiction is better revealed by Figures 4(h) and (i), in which the two optical flares appear very different in brightness and duration while the X-ray profiles are nearly identical. It is also unclear how the absorbing gas becomes so clumpy as to form cloud gaps very frequently. Taken as a whole the variable absorption scenario is unable to explain the flaring state in many ways, and so we rule the possibility out.

\subsection{Thermal Scenarios for the Soft X-Ray Excess}

As demonstrated in Section 1, the soft X-ray excess of the saddle-shaped spectra can be modeled by a single-temperature blackbody (a multi-temperature disk has been ruled out as the inferred inner radius is too small). If this thermal component is genuine, the neutron star surface will be the most reasonable origin of the emission. Though it is still unclear whether tMSPs are accretion-powered (e.g., Bogdanov et al. 2015; Papitto \& Torres 2015) or rotation-powered (e.g., Takata et al. 2014; Jaodand et al. 2016; Ambrosino et al. 2017; Papitto et al. 2019) during the sub-luminous disk state, such thermal emission is actually possible in both cases.

For accretion-powered pulsars, hotspots can be formed on the neutron star surface when the magnetically channelled 
accretion flows heat up the magnetic poles. The temperatures of the hotspots are around $0.1-1 \mathrm{keV}$, which are consistent with the thermal component (blackbody or nsa) of 3FGL J0427.9 -6704 . However, the apparent size of the blackbody (i.e., $\sim 0.1 \mathrm{~km}$; Table 1) is much smaller than the typical size of the hotspots seen in AMXPs (i.e., radius of a few km; Gierliński et al. 2002; Gierliński \& Poutanen 2005) and tMSPs (e.g., $\approx 3 \mathrm{~km}$ for PSR J1023+0038 in the high mode; Bogdanov et al. 2015). For the nsa fits, despite the larger emission sizes inferred, most of them are still less than $1 \mathrm{~km}$. Mediuml is the only data set that yields an emission region larger than $1 \mathrm{~km}$, but the statistical uncertainties are also huge, making the case marginal.

For rotation-powered pulsars, X-ray-emitting regions with temperatures of $0.1-1 \mathrm{keV}$ can be created by polar cap heating (see Harding \& Muslimov 2002 and references therein). These heated polar cap regions are expected to be large $(\sim 1 \mathrm{~km}$ in radius for MSPs), which is, again, too big for the thermal component of 3FGL J0427.9-6704. Alternatively, the thermal $\mathrm{X}$-rays could be generated by the back-flow of the primary charged particles from the outer gap. The heated region would be much smaller in this scenario (i.e., $\sim 0.1 \mathrm{~km}$ in radius; Zhang $\&$ Cheng 2003). However, such thermal components should be accompanied by another, slightly cooler (i.e., $\lesssim 0.1 \mathrm{keV}$ ), but larger (i.e., $\sim 1 \mathrm{~km}$ ) blackbody component, which is not seen in 3FGL J0427.9-6704.

Apart from the size inconsistency, the weak X-ray absorption for the thermal component is unexplainable. If the soft $\mathrm{X}$-ray photons are really coming from the pulsar surface (the innermost observable region of the system), the thermal component should be highly absorbed. However, no intrinsic absorption is observed for the thermal emission, in contrast to the strong intrinsic absorption found for the nonthermal component. Besides, the inferred photon indices of the nonthermal component are significantly harder $(\Gamma \approx 0.9-1.1)$ than those of the two known tMSPs in the sub-luminous state ( $\Gamma \approx 1.6-1.8$; de Martino et al. 2013; Li et al. 2014; Bogdanov et al. 2015), while the partially absorbed power law gives more reasonable results in this sense, especially in Lowl and Mediuml ( $\Gamma \approx 1.8$ and 1.6, respectively; Table 1$)$.

Based on the above arguments, we conclude that an extra thermal component as the origin for the soft X-ray excess is highly unlikely.

\subsection{Nonthermal Scenarios}

For a more physical picture, we considered the "propeller" scenario (Illarionov \& Sunyaev 1975), which has been widely used to understand the mode-switching phenomenon as well as the high-energy emission observed in tMSPs (Archibald et al. 2015; Papitto \& Torres 2015; Campana et al. 2016). In the socalled propeller regime, where the accretion disk is truncated by the pulsar magnetosphere outside the co-rotation radius (i.e., $r_{\mathrm{m}}>r_{\mathrm{c}}$ ), most of the inflowing material is ejected by the centrifugal barrier, but a small fraction of the gas can still be accreted onto the neutron star through the magnetic field (D’Angelo et al. 2015). Recent magnetohydrodynamics simulations have shown that this partial accretion process can be possible for neutron star systems (Lii et al. 2014). The X-ray pulsations of PSR J1023+0038 and PSR J1227-4853 detected in the high mode (Archibald et al. 2015; Papitto et al. 2015) could be evidence for this partial accretion.
As mentioned in Section 1, the low, high, and flare accretion modes are common in PSRs J1023+0038 and J1227-4853. In Figure 2, we compare the X-ray light curve of 3FGL J0427.9 -6704 with the X-ray luminosities of PSR J1023+0038 in the three modes (Bogdanov et al. 2015). The "quiescent" state of 3FGL J0427.9-6704 has a similar luminosity to the low mode of PSR J1023+0038. No obvious high mode of 3FGL J0427.9 -6704 is seen in the XMM-Newton light curve, but some weak X-ray flares are comparable to the high-mode flux of PSR J1023+0038 (e.g., the fourth X-ray flare just after $t=2500 \mathrm{~s}$ in Figure 2).

One possibility is that these weak flares represent a transitory high mode in 3FGL J0427.9-6704. It has been suggested that the high and low modes are referring to a tMSP system staying in or away from the propeller regime, respectively (Campana et al. 2016). In this context, 3FGL J0427.9-6704 might stay in the propeller regime with a short duration of $\sim 10-40 \mathrm{~s}$ in typical flaring episodes. This would also be in agreement with the likely small inner disk inferred in S16 (an inner radius of a few tens of kilometers, which is comparable to the co-rotation radius of an MSP). A relatively weak and/or unstable accretion flow of 3FGL J0427.9-6704, which could not support a sustainable propeller state, would be a possible reason for the transitory high mode.

There are numerous possible counterarguments to this picture: the concurrent X-ray and optical/UV variability of 3FGL J0427.9-6704 is quite unlike that for PSR J1023 +0038 , where the optical flux has not been observed to follow the low/high mode switching (Bogdanov et al. 2015). Optical variability that is analogous to the X-ray mode switching was found in the light curves of PSR J1023+0038 though (Shahbaz et al. 2015, 2018; Hakala \& Kajava 2018). In addition, a negative correlation is seen between the radio and X-ray luminosities in the low and high modes of PSR J1023+0038 (Bogdanov et al. 2018), but the correlation is likely positive in 3FGL J0427.9-6704. The $N_{\mathrm{H}}$ variation could be another issue: strong absorption is generally expected if the X-ray emission originates from a more inner region in the high mode, but 3FGL J0427.9-6704 shows the opposite. Further, the partial $\mathrm{X}$-ray absorption would be hard to explain, if the emission region is tiny (a few tens of kilometers) - the emission would likely be "fully" absorbed.

Alternatively, the absence of the high mode in 3FGL J0427.9-6704 could also mean that the system did not enter the propeller regime at all. Provided that the rotation-powered activity of the radio/ $\gamma$-ray pulsar turned on to push the inner edge of the disk away from the light cylinder during the entire XMM-Newton observation, the emission coming from the intrabinary shock between the relativistic pulsar wind and the accretion flow would take over in the X-ray band ( $\mathrm{Li}$ et al. 2014; Takata et al. 2014; Campana et al. 2016). Perturbation of the shock front due to the instability in the accretion flow can produce X-ray variability on a timescale of $\sim 100 \mathrm{~s}$ (Takata et al. 2014), which could be the origin of the flares. The concurrent optical/UV variability could then be attributed to the instability of the accretion disk that triggers the X-ray flares. Depending on the momentum ratio between the accretion flow and the pulsar wind, the intrabinary shock radius of PSR J1023 +0038 could be $\sim 10^{10}-10^{11} \mathrm{~cm}$ during the sub-luminous disk state (a few thousand times larger than the co-rotation radius of an MSP; Li et al. 2014; Takata et al. 2014). If 3FGL J0427.9-6704 


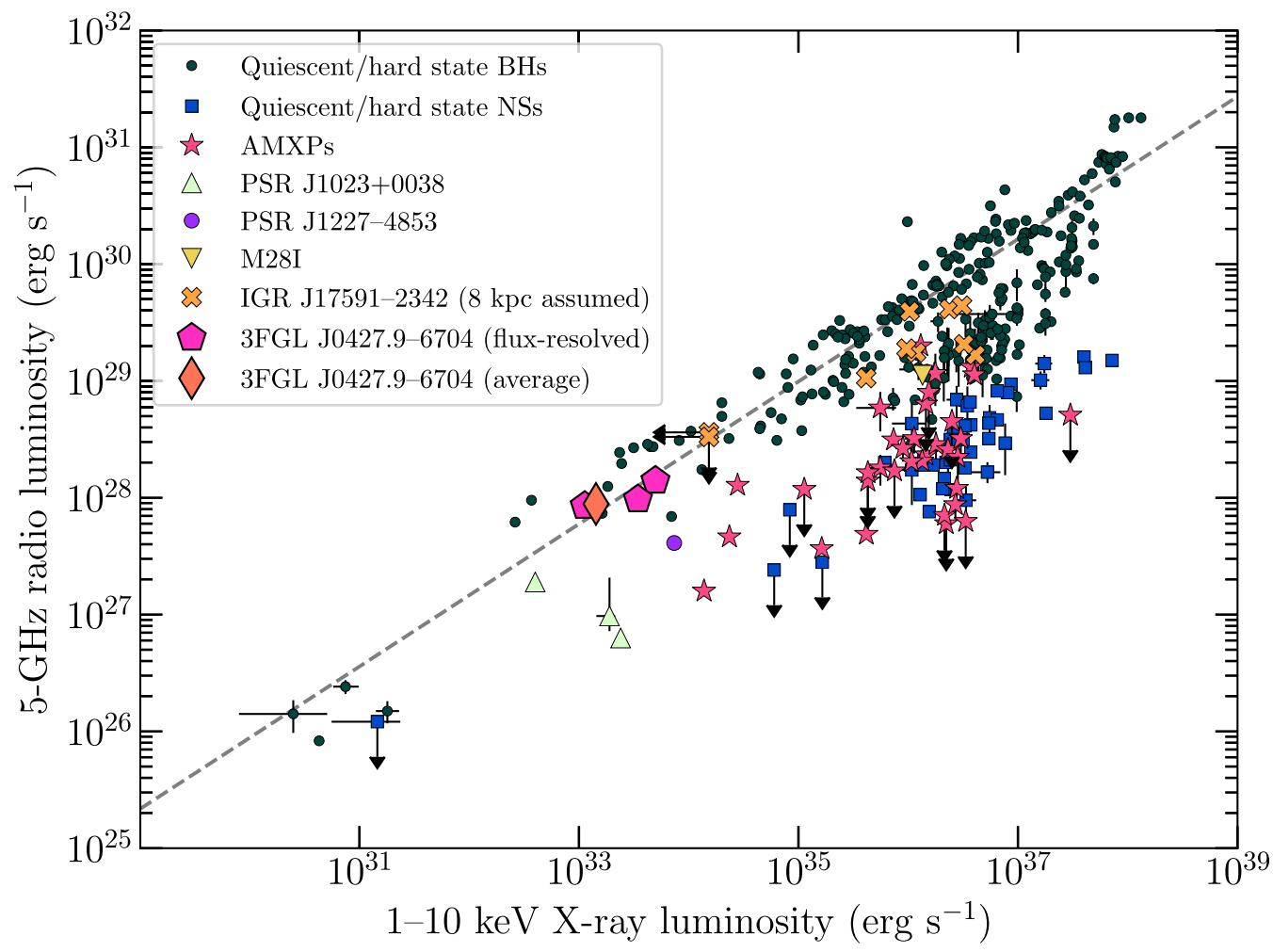

Figure 10. $L_{X}-L_{R}$ plot containing 3FGL J0427.9-6704, IGR J17591-2342 (Gusinskaia et al. 2020), three tMSPs, and other low-mass X-ray binaries obtained from the database of Bahramian et al. (2018). 3FGL J0427.9-6704 sits close to the black hole relation at $L_{X} \sim 10^{33} \mathrm{erg} \mathrm{s}^{-1}$ and is the only low- $L_{X}$ neutron star yet observed to do so.

has a similar shock size, the partial X-ray absorption feature can be understood more easily. However, the large shock requires an inner disk radius of $\sim 10^{9} \mathrm{~cm}$ ( $\mathrm{Li}$ et al. 2014), which is inconsistent with the result of S16 based on the optical lightcurve modeling. The problem regarding the $N_{\mathrm{H}}$ variation also stays unsolved in this theoretical frame.

Models have also posited different explanations for the (less frequent) flares observed in other tMSPs. For example, in the tMSP model of Veledina et al. (2019), flares are caused by temporary increases in the cross-section of the pulsar wind/disk interaction. However, the origin of these variations is not obvious and does not give a straightforward prediction for the frequency or length of the flares observed in 3FGL J0427.9-6704.

\subsection{Radio-X-Ray Correlation}

Accreting black holes show nearly ubiquitous radio continuum emission in their low/hard states, usually associated with a partially self-absorbed compact jet. However, our understanding of radio emission from accreting neutron stars has been slower to develop, with evidence emerging in the last decade of a much more complex situation for neutron stars than for black holes. It is clear that neutron stars do not follow a single relation between radio and X-ray luminosity, but show a wide range of radio-loudness at all $L_{X}>10^{34} \mathrm{erg} \mathrm{s}^{-1}$ (e.g., Migliari \& Fender 2006; Migliari et al. 2011; Tudor et al. 2017; Gallo et al. 2018; Gusinskaia et al. 2020).

The radio/X-ray correlation for neutron stars at a range of $L_{X}$ can help distinguish among physical models for the accretion flow. It also bears on the practice of using the radio-loudness of an accreting compact object to distinguish the nature of the accretor-typically neutron star versus black hole-at both low (e.g., Strader et al. 2012) and high (e.g., Ludlam et al. 2019) $\mathrm{X}$-ray luminosity.

Figure 10 shows the radio/X-ray correlation for known black holes and neutron stars. Previous radio continuum studies of tMSPs have shown that these systems reliably show radio emission in the sub-luminous disk state (Hill et al. 2011; Papitto et al. 2013; Deller et al. 2015; Jaodand 2019). These few published tMSPs appear to sit on a track which is parallel to the black hole radio/X-ray correlation, but a factor of few fainter.

Remarkably, 3FGL J0427.9-6704 sits directly on the black hole correlation rather than the tMSP correlation. Since its radio continuum flux density depends only weakly on X-ray luminosity, this statement is true both in the time-averaged sense and for subsets of the data selected by X-ray luminosity. The robustness of its location is unlike PSR $\mathrm{J} 1023+0038$, where the mean radio/X-ray ratio is consistent with the proposed tMSP correlation, but the inverse behavior of radio and X-ray during mode switching means that the source is closer to the black hole correlation in the low mode, but closer to an extension of a "hard state" neutron star correlation in the X-ray high mode (Figure 10).

The only other accreting neutron star shown to sit close to the black hole radio/X-ray correlation is the AMXP IGR $\mathrm{J} 17591-2342$, but this is at much higher $L_{X} \gtrsim 10^{35} \mathrm{erg} \mathrm{s}^{-1}$, and its distance is also not yet well-constrained (Gusinskaia et al. 2020). This paper points out that there is no obvious reason why this AMXP should be much more radio-loud than other similar systems, and some candidate explanations such as the spin rate of the neutron star or possible beaming are not consistent with the data. 
While the radio continuum emission in the sub-luminous disk state of PSR J1023+0038 was initially mooted as arising in a jet (Deller et al. 2015), the extreme radio variability observed on short timescales led Bogdanov et al. (2018) to conclude that a steady jet could not be present. Instead, they suggest the radio emission could arise from expanding plasma bubbles at the interface of the pulsar magnetosphere and the inner disk.

By contrast, the radio emission from 3FGL J0427.9-6704 has properties more consistent with jets observed for black holes of a similar $L_{X}$ : it is comparably radio-luminous, has a flat spectrum, is of substantial spatial extent $\left(>>3.5 \times 10^{10} \mathrm{~cm}\right)$, and is relatively stable on timescales of hours to months. While these properties do not prove that the radio emission arises from a jet rather than some other sort of sustained outflow, they are consistent with what one would expect for a jet.

\subsection{Other Flare-dominated Sources?}

Here we briefly discuss another system that shares some properties with 3FGL J0427.9-6704. In the globular cluster NGC 6652, the second-brightest source (here referred to as NGC 6652B) has $L_{X} \sim 10^{34} \mathrm{erg} \mathrm{s}^{-1}$ and shows flare-like variability on timescales of a few $\times 100 \mathrm{~s}$ in a $201147 \mathrm{ks}$ Chandra observation (Stacey et al. 2012). Optical photometry of the source also displays rapid variability (Engel et al. 2012). While NGC 6652B is undoubtedly more luminous than 3FGL J0427.9-6704, the phenomenology is sufficiently similar to be worthy of further study.

\section{Conclusion}

The observed properties of our simultaneous XMM-Newton and ATCA observations of the edge-on tMSP candidate, 3FGL J0427.9-6704 are summarized as follows.

1. The X-ray variability seen in the 2016 NuSTAR observation is resolved by XMM-Newton EPIC. The variability is caused by vigorous X-ray flaring of the LMXB. While the flare mode accretion is occasionally seen in PSRs J1023+0038 and J1227-4853, the accretion state of 3FGL J0427.9-6704 is entirely flaredominated at a high flare occurrence rate of $\sim 2 \mathrm{ks}^{-1}$. As the flares disappear during the three pulsar eclipses (Figure 2), we conclude that the flares originate from the accreting neutron star. Except for the eclipses, the flares do not show any orbital dependence (Figure 5).

2. The flares are observed simultaneously in X-rays and optical/UV by XMM-Newton EPIC and OM. Almost all of the X-ray flares have a corresponding optical/UV counterpart, but not every optical/UV flare has an X-ray partner. No significant time offset is seen between the X-ray flares and their optical/UV counterparts, with a formal crosscorrelation offset of $4.5 \pm 6.8 \mathrm{~s}$ (Figure 6). For those paired up, the flare durations in optical/UV are longer than those in X-rays, and the flare amplitudes are only weakly correlated (Figure 7). Nevertheless, the optical/UV emission appears to lead the X-ray emission in a few cases (e.g., the flares shown in Figures 4(h), (i), and (p)).

3. The X-ray spectra (average or flux-resolved) of 3FGL J0427.9-6704 are saddle-like with a significant soft X-ray bump below $2 \mathrm{keV}$ (Figure 1). This feature can be modeled either by an ordinary absorbed power law with an additional thermal component or a partially absorbed power law (Table 1). We ruled out multicomponent models because of the unphysically small thermal emission size inferred and/or the absence of intrinsic absorption for the thermal X-rays. In the fluxresolved analysis with a partially absorbed power law, 3FGL J0427.9-6704 becomes spectrally harder (i.e., lower $\Gamma$ ) with lower $N_{\mathrm{H}}$ at higher luminosities. There could be a high-energy exponential cut-off at $E_{\mathrm{c}} \approx$ $30 \mathrm{keV}$ based on the joint XMM-Newton-NuSTAR spectrum, but this conclusion is tentative as the two $\mathrm{X}$-ray data sets were non-simultaneous.

4. We detect weak X-ray emission during the three X-ray eclipses, possibly due to X-ray scattering off the companion's atmosphere.

5. We consider the X-ray and optical properties of 3FGL J0427.9-6704 in the context of the commonly discussed propeller and intrabinary shock scenarios for the known tMSPs, but find that none of these models does a great job at explaining the observed phenomenology.

6. Mostly steady radio continuum emission is seen in all our ATCA observations, and we find that the radio flux density is positively correlated with the X-ray luminosity. Unlike for the optical and X-ray, we observe no radio eclipses. The stable, spatially extended, flat-spectrum radio emission has properties consistent with a jet.

7. 3FGL J0427.9-6704 sits precisely on the black hole radio/ X-ray correlation, proving that even at $L_{X} \sim 10^{33} \mathrm{erg} \mathrm{s}^{-1}$, some neutron stars can be as radio-bright as black holes.

We acknowledge useful discussions with K. Sokolovsky. This work was supported by NASA grant 80NSSC18K0382, NSF grant AST-1714825, and a Packard Fellowship. K.L.L. is supported by the Ministry of Science and Technology of the Republic of China (Taiwan) through grant 108-2112-M-007-025MY3. J.C.A.M.-J. is the recipient of an Australian Research Council Future Fellowship, funded by the Australian government. $\mathrm{COH}$ is funded by NSERC Discovery Grant RGPIN-2016-04602. This work is based on observations obtained with XMM-Newton an ESA science mission with instruments and contributions directly funded by ESA Member States and NASA. The Australia Telescope Compact Array is part of the Australia Telescope National Facility which is funded by the Australian Government for operation as a National Facility managed by CSIRO.

Facilities: XMM, ATCA.

\section{ORCID iDs}

Kwan-Lok Li (D) https:// orcid.org/0000-0002-0439-7047

Jay Strader (i) https://orcid.org/0000-0002-1468-9668 James C. A. Miller-Jones (i) https://orcid.org/0000-00033124-2814

Craig O. Heinke (D) https://orcid.org/0000-0003-3944-6109

Laura Chomiuk (iD https://orcid.org/0000-0002-8400-3705

\section{References}

Abdollahi, S., Acero, F., Ackermann, M., et al. 2020, ApJS, 247, 33 Alpar, M. A., Cheng, A. F., Ruderman, M. A., \& Shaham, J. 1982, Natur, 300,728

Ambrosino, F., Papitto, A., Stella, L., et al. 2017, NatAs, 1, 854

An, H., Romani, R. W., Johnson, T., Kerr, M., \& Clark, C. J. 2017, ApJ, 850,100

Archibald, A. M., Bogdanov, S., Patruno, A., et al. 2015, ApJ, 807, 62

Archibald, A. M., Stairs, I. H., Ransom, S. M., et al. 2009, Sci, 324, 1411

Avni, Y. 1976, ApJ, 210, 642 
Bahramian, A., Miller-Jones, J., Strader, J., et al. 2018, Radio/X-Ray Correlation Database for X-ray Binaries, https://github.com/bersavosh/ XRB-LrLx_pub

Bailer-Jones, C. A. L., Rybizki, J., Fouesneau, M., Mantelet, G., \& Andrae, R. 2018, AJ, 156, 58

Bogdanov, S., Archibald, A. M., Bassa, C., et al. 2015, ApJ, 806, 148

Bogdanov, S., Deller, A. T., Miller-Jones, J. C. A., et al. 2018, ApJ, 856, 54

Bogdanov, S., \& Halpern, J. P. 2015, ApJL, 803, L27

Bogdanov, S., Heinke, C. O., Özel, F., \& Güver, T. 2016, ApJ, 831, 184

Bond, H. E., White, R. L., Becker, R. H., \& O'Brien, M. S. 2002, PASP, 114, 1359

Britt, C. T., Strader, J., Chomiuk, L., et al. 2017, ApJ, 849, 21

Campana, S., Coti Zelati, F., Papitto, A., et al. 2016, A\&A, 594, A31

Cash, W. 1979, ApJ, 228, 939

Cho, P. B., Halpern, J. P., \& Bogdanov, S. 2018, ApJ, 866, 71

Church, M. J., \& Bałucińska-Church, M. 2004, MNRAS, 348, 955

Coti Zelati, F., Papitto, A., de Martino, D., et al. 2019, A\&A, 622, A211

D’Angelo, C. R., Fridriksson, J. K., Messenger, C., \& Patruno, A. 2015, MNRAS, 449, 2803

de Martino, D., Belloni, T., Falanga, M., et al. 2013, A\&A, 550, A89

Deller, A. T., Moldon, J., Miller-Jones, J. C. A., et al. 2015, ApJ, 809, 13

Engel, M. C., Heinke, C. O., Sivakoff, G. R., Elshamouty, K. G., \& Edmonds, P. D. 2012, ApJ, 747, 119

Gallo, E., Degenaar, N., \& van den Eijnden, J. 2018, MNRAS, 478, L132

Gierliński, M., Done, C., \& Barret, D. 2002, MNRAS, 331, 141

Gierliński, M., \& Poutanen, J. 2005, MNRAS, 359, 1261

Gusinskaia, N. V., Russell, T. D., Hessels, J. W. T., et al. 2020, MNRAS, 492, 1091

Hakala, P., \& Kajava, J. J. E. 2018, MNRAS, 474, 3297

Halpern, J. P., Strader, J., \& Li, M. 2017, ApJ, 844, 150

Harding, A. K., \& Muslimov, A. G. 2002, ApJ, 568, 862

Heinke, C. O., Grindlay, J. E., Lloyd, D. A., \& Edmonds, P. D. 2003, ApJ, 588,452

Hill, A. B., Szostek, A., Corbel, S., et al. 2011, MNRAS, 415, 235

Hui, C. Y., \& Li, K. L. 2019, Galax, 7, 93

Illarionov, A. F., \& Sunyaev, R. A. 1975, A\&A, 39, 185

Jaodand, A. 2019, PhD thesis, Netherlands Inst. of Radio Astronomy (ASTRON) and Univ. Amsterdam

Jaodand, A., Archibald, A. M., Hessels, J. W. T., et al. 2016, ApJ, 830, 122

Jaodand, A., Hessels, J. W. T., \& Archibald, A. 2018, in IAU Symp. 337,

Pulsar Astrophysics the Next Fifty Years, ed. P. Weltevrede et al. (Cambridge: Cambridge Univ. Press), 47

Johnson, T. J., Ray, P. S., Roy, J., et al. 2015, ApJ, 806, 91

Kalberla, P. M. W., Burton, W. B., Hartmann, D., et al. 2005, A\&A, 440, 775
Kennedy, M. R., Clark, C. J., Voisin, G., \& Breton, R. P. 2018, MNRAS, 477,1120

Lee, J., Hui, C. Y., Takata, J., et al. 2018, ApJ, 864, 23

Li, K. L., Kong, A. K. H., Takata, J., et al. 2014, ApJ, 797, 111

Lii, P. S., Romanova, M. M., Ustyugova, G. V., Koldoba, A. V., \& Lovelace, R. V. E. 2014, MNRAS, 441, 86

Ludlam, R. M., Shishkovsky, L., Bult, P. M., et al. 2019, ApJ, 883, 39

McMullin, J. P., Waters, B., Schiebel, D., Young, W., \& Golap, K. 2007, in ASP Conf. Ser. 376, Astronomical Data Analysis Software and Systems XVI, ed. R. A. Shaw, F. Hill, \& D. J. Bell (San Francisco, CA: ASP), 127 Migliari, S., \& Fender, R. P. 2006, MNRAS, 366, 79

Migliari, S., Miller-Jones, J. C. A., \& Russell, D. M. 2011, MNRAS, 415, 2407

Papitto, A., Ambrosino, F., Stella, L., et al. 2019, ApJ, 882, 104

Papitto, A., de Martino, D., Belloni, T. M., et al. 2015, MNRAS, 449, L26

Papitto, A., Ferrigno, C., Bozzo, E., et al. 2013, Natur, 501, 517

Papitto, A., Rea, N., Coti Zelati, F., et al. 2018, ApJL, 858, L12

Papitto, A., \& Torres, D. F. 2015, ApJ, 807, 33

Patruno, A., Archibald, A. M., Hessels, J. W. T., et al. 2014, ApJL, 781, L3

Radhakrishnan, V., \& Srinivasan, G. 1982, CSci, 51, 1096

Romani, R. W. 2012, ApJL, 754, L25

Romani, R. W., Filippenko, A. V., \& Cenko, S. B. 2015, ApJ, 804, 115

Roy, J., Ray, P. S., Bhattacharyya, B., et al. 2015, ApJL, 800, L12

Shahbaz, T., Dallilar, Y., Garner, A., et al. 2018, MNRAS, 477, 566

Shahbaz, T., Linares, M., Nevado, S. P., et al. 2015, MNRAS, 453, 3461

Stacey, W. S., Heinke, C. O., Cohn, H. N., Lugger, P. M., \& Bahramian, A. 2012, ApJ, 751, 62

Standish, E. M., Jr. 1990, A\&A, 233, 252

Stappers, B. W., Archibald, A. M., Hessels, J. W. T., et al. 2014, ApJ, 790, 39

Strader, J., Chomiuk, L., Maccarone, T. J., Miller-Jones, J. C. A., \& Seth, A. C. 2012, Natur, 490, 71

Strader, J., Li, K.-L., Chomiuk, L., et al. 2016, ApJ, 831, 89

Strader, J., Swihart, S., Chomiuk, L., et al. 2019, ApJ, 872, 42

Takata, J., Li, K. L., Leung, G. C. K., et al. 2014, ApJ, 785, 131

Tendulkar, S. P., Yang, C., An, H., et al. 2014, ApJ, 791, 77

Thorstensen, J. R., \& Armstrong, E. 2005, AJ, 130, 759

Tudor, V., Miller-Jones, J. C. A., Patruno, A., et al. 2017, MNRAS, 470, 324

Veledina, A., Nättilä, J., \& Beloborodov, A. M. 2019, ApJ, 884, 144

Wang, Z., Archibald, A. M., Thorstensen, J. R., et al. 2009, ApJ, 703, 2017

White, N. E., \& Holt, S. S. 1982, ApJ, 257, 318

Wilson, W. E., Ferris, R. H., Axtens, P., et al. 2011, MNRAS, 416, 832

Yap, Y. X., Li, K. L., Kong, A. K. H., et al. 2019, A\&A, 621, L9

Zhang, L., \& Cheng, K. S. 2003, A\&A, 398, 639 